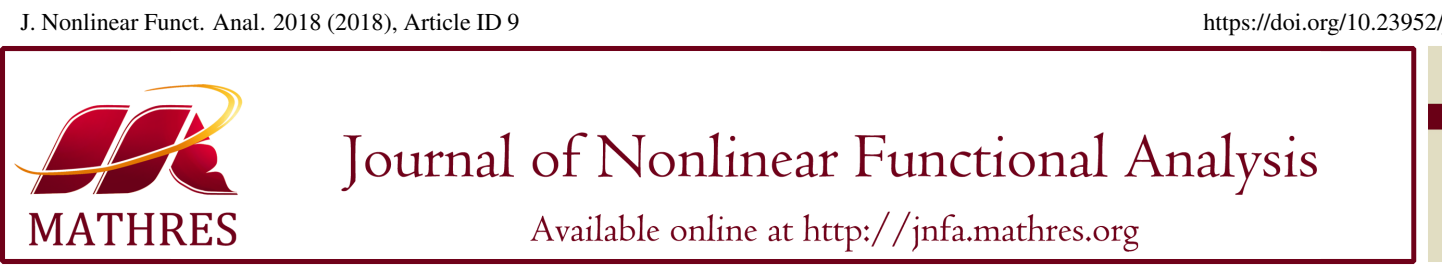

https://doi.org/10.23952/jnfa.2018.9

\title{
EXISTENCE RESULTS AND THE MONOTONE ITERATIVE TECHNIQUES FOR SYSTEMS OF NONLINEAR FRACTIONAL DIFFERENTIAL EQUATIONS WITH INTEGRAL BOUNDARY CONDITIONS
}

\author{
MOHAMMED DERHAB*, FATIHA MEZIANE \\ Department of Mathematics, Faculty of Sciences, Abou-Bekr Belkaid Tlemcen University, B.P.119, Tlemcen 13000, Algeria
}

\begin{abstract}
In this paper, based on the upper and lower solutions method and monotone iterative techniques, we study the existence of solutions for fractional differential systems with continuous nonlinearities and integral boundary conditions. The construction of the monotone sequences and the definition of upper and lower solutions depend on the quasimonotone property of the reaction functions. We prove the existence of maximal and minimal solutions for quasimonotone increasing systems. Also, we prove the existence of maximal-minimal and minimal-maximal solutions for quasimonotone decreasing systems and for mixed quasimonotone systems and the existence of at least one solution. Finally, we give some examples to illustrate our results.

Keywords. Riemann-Liouville fractional derivative; Fractional differential systems; Integral boundary condition; Upper and lower solutions; Monotone iterative technique.
\end{abstract}

2010 Mathematics Subject Classification. 34A08, 34A12, 34B15.

\section{INTRODUCTION}

The purpose of this paper is to study the existence of solutions for a class of first order fractional differential systems subject to integral boundary conditions. More precisely, we consider the following nonlinear boundary value problem

$$
\left\{\begin{array}{l}
D^{\alpha_{1}} u(t)=f(t, u, v), t \in(0,1], \\
D^{\alpha_{2}} v(t)=g(t, u, v), t \in(0,1], \\
\left.t^{1-\alpha_{1}} u(t)\right|_{t=0}=\int_{0}^{1} g_{1}(s) u(s) d s, \\
\left.t^{1-\alpha_{2}} v(t)\right|_{t=0}=\int_{0}^{1} g_{2}(s) v(s) d s,
\end{array}\right.
$$

where $D^{\alpha_{i}}$ is the Riemann-Liouville fractional derivative of order $\alpha_{i}$ with $0<\alpha_{i}<1$ for $i=1,2, f$ : $(0,1] \times \mathbb{R}^{2} \rightarrow \mathbb{R}, g:(0,1] \times \mathbb{R}^{2} \rightarrow \mathbb{R}$ and $g_{i}:[0,1] \rightarrow \mathbb{R}_{+}(i=1,2)$ are continuous functions.

${ }^{*}$ Corresponding author.

E-mail addresses: derhab@yahoo.fr (M. Derhab), tihameziane@ hotmail.fr (F. Meziane).

Received June 16, 2017; Accepted February 12, 2018.

(C)2018 Journal of Nonlinear Functional Analysis 
Fractional differential equations or fractional differential systems arise in many scientific fields such as plasticity, viscoelasticity, electrical circuits, electroanalytical chemistry, biology, control theory, pharmacodynamics, pharmacokinetics, electromagnetic theory, biomedical problems, psychological and life sciences; see [1], [2], [3], [4], [5], [6] and the references therein. Fractional differential systems with nonlocal boundary conditions have been studied by several authors using the upper and lower solutions method, the monotone iterative methods and fixed point theorems in cones; see [7], [8], [9], [10] and the references therein.

It is well know that the method of upper and lower solutions coupled with monotone iterative techniques has been used to prove existence of solutions of nonlinear boundary value problems by various authors; see [10], [11], [12], [13], [14], [15], [16] and [17]. Observe that the definition itself of upper and lower solutions of (1.1) and the construction of the monotone sequences depend on the quasimonotone property of the reaction functions $f$ and $g$. Thus, following Pao's notation in [16], we can classify (1.1) according to their relative monotony as follows:

Type 1. Quasimonotone increasing systems: $f$ is increasing in $v$ and $g$ in $u$.

Type 2. Quasimonotone decreasing systems: $f$ is decreasing in $v$ and $g$ in $u$.

Type 3. Mixed quasimonotone systems: $f$ is increasing in $v$ and $g$ is decreasing in $u$ or vice versa.

In this paper, we prove the existence of maximal and minimal solutions for systems of Type 1 . These solutions are the limits of two monotone sequences. Also, we prove the existence of maximal-minimal and minimal-maximal solutions for systems of Type 2. When the system is of type 3 and if $\alpha_{1}=\alpha_{2}$, we prove the existence of at least one solution. Our results improve and generalize the results obtained in [10], [13] and [17]. We note also that to the best of our knowledge this is the first paper which uses the method of upper and lower solutions coupled with monotone iterative techniques to prove the existence of solutions for mixed quasimonotone systems with integral boundary conditions.

The plan of this paper is as follows: In Section 2, we give some preliminary results that will be used throughout the paper. In Section 3, we study the existence of maximal and minimal solutions for quasimonotone increasing systems. Section 4 is concerned with the existence of maximal-minimal and minimal-maximal solutions for quasimonotone decreasing systems. In Section 5, we study the existence of solutions for mixed quasimonotone systems. Finally in Section 6, we give some examples to illustrate our results.

\section{PRELIMINARIES}

In this section, we give some definitions and preliminary results that will be used in the remainder of this paper.

Definition 2.1. Let $0<q<1$. We denote by $C_{1-q}([0,1])$, the function space

$$
C_{1-q}([0,1])=\left\{u \in C((0,1]) ; t^{1-q} u(t) \in C([0,1])\right\} .
$$

Definition 2.2. For $u \in C_{1-q}([0,1])$, we define the weighted norm by

$$
\|u\|=\max _{t \in[0,1]}\left|t^{1-q} u(t)\right| .
$$

Remark 2.3. $\left(C_{1-q}([0,1]),\|\cdot\|\right)$ is a Banach space. 
Definition 2.4. Let $0<q<1$ and let $h \in C_{1-q}([0,1])$. The Riemann-Liouville integral of order $q$ of $h$ is defined by

$$
I^{q} h(t):=\frac{1}{\Gamma(q)} \int_{0}^{t}(t-s)^{q-1} h(s) d s,
$$

where $\Gamma$ is the Gamma Euler function defined by

$$
\Gamma(z)=\int_{0}^{+\infty} \exp (-t) t^{z-1} d z,
$$

where $z \in \mathbb{C}$ with $\mathfrak{R}(z)>0$.

Definition 2.5. Let $0<q<1$ and let $h \in C_{1-q}([0,1])$. The Riemann-Liouville fractional derivative of order $q$ of $h$ is defined by $D^{q} h(t)=\frac{d}{d t} I^{1-q} h(t)$.

Now, we consider this following initial problem

$$
\left\{\begin{array}{l}
D^{q} u(t)+M u(t)=\widetilde{h}(t), t \in(0,1], \\
\left.t^{1-q} u(t)\right|_{t=0}=\widetilde{u_{0}}
\end{array}\right.
$$

where $0<q<1, M$ is a positive constant, $\widetilde{h} \in C_{1-q}([0,1])$ and $\widetilde{u_{0}} \in \mathbb{R}$.

We have the following results.

Lemma 2.6. [5] Problem (2.1) admits a unique solution $u$ which is given by

$$
u(t)=\Gamma(q) \widetilde{u_{0}} t^{q-1} E_{q, q}\left(-M t^{q}\right)+\int_{0}^{t}(t-s)^{q-1} E_{q, q}\left(-M(t-s)^{q}\right) \widetilde{h}(s) d s,
$$

where $E_{q, q}$ is the Mittag-Leffler function defined by

$$
E_{q, q}(z)=\sum_{n=0}^{\infty} \frac{z^{n}}{\Gamma(q(n+1))}, z \in \mathbb{C}
$$

Remark 2.7. If $u$ is a solution of (2.1), then $u \in C_{1-q}([0,1])$.

Now, for $0<q<1$, we define the Mittag-Leffler function $E_{q}$ by

$$
E_{q}(z)=\sum_{n=0}^{\infty} \frac{z^{n}}{\Gamma(q n+1)}, z \in \mathbb{C} .
$$

We have the following result.

Theorem 2.8. [18, Theorem 4.2.] For $0<q<1, E_{q}$ has no zeros on the real axis; $0<E_{q}(x)<1$ for $x<0$ and $\frac{d}{d x} E_{q}(x)>0$ for the whole real axis.

Lemma 2.9. For $0<q<1$, one has

(i) $E_{q, q}(-x)=-q \frac{d}{d x} E_{q}(-x)$, for $x \geq 0$,

(ii) $E_{q, q}(-x)>0$, for $x \geq 0$.

Proof. Letting $0<q<1$ and $x \geq 0$, we have 
(i)

$$
\begin{aligned}
\frac{d}{d x} E_{q}(-x) & =\frac{d}{d x} \sum_{n=0}^{+\infty} \frac{(-x)^{n}}{\Gamma(q n+1)} \\
& =\sum_{n=1}^{+\infty} \frac{-n(-x)^{n-1}}{\Gamma(q n+1)} \\
& =\sum_{n=1}^{+\infty} \frac{-n(-x)^{n-1}}{n q \Gamma(q n)} \\
& =\frac{-1}{q} \sum_{n=0}^{+\infty} \frac{(-x)^{n}}{\Gamma(q(n+1))} \\
& =\frac{-E_{q, q}(-x)}{q} .
\end{aligned}
$$

Then

(ii) By Theorem 2.8, we have

$$
E_{q, q}(-x)=-q \frac{d}{d x} E_{q}(-x)
$$

$$
\frac{d}{d x} E_{q}(-x)=-\frac{d}{d(-x)} E_{q}(-x)<0 .
$$

Using (i), one finds that $E_{q, q}(-x)>0$.

Lemma 2.10. Let $u \in C_{1-q}([0,1])$ with $0<q<1$ and assume that $u$ satisfies

$$
\left\{\begin{array}{l}
D^{q} u(t)+M u(t) \geq 0, t \in(0,1] \\
\left.t^{1-q} u(t)\right|_{t=0} \geq 0
\end{array}\right.
$$

Then $t^{1-q} u(t) \geq 0$ for all $t \in[0,1]$.

Proof. The proof of this Lemma is a consequence of Lemma 2.6 and (ii) of Lemma 2.9.

Lemma 2.11. Let $u \in C_{1-q}([0,1])$ with $0<q<1$ and assume that $u$ satisfies

$$
\left\{\begin{array}{l}
D^{q} u(t)+\widetilde{M} u(t) \leq 0, t \in(0,1], \\
\left.t^{1-q} u(t)\right|_{t=0} \leq \int_{0}^{1} \widetilde{g}(s) u(s) d s
\end{array}\right.
$$

where $\widetilde{M} \leq 0$ and $\widetilde{g}:[0,1] \rightarrow \mathbb{R}_{+}$is a continuous function such that

$$
\Gamma(q) \int_{0}^{1} s^{q-1} E_{q, q}\left(-\widetilde{M} s^{q}\right) \widetilde{g}(s) d s<1 .
$$

Then $t^{1-q} u(t) \leq 0$ for all $t \in[0,1]$.

Proof. Let $u \in C_{1-q}([0,1])$ with $0<q<1$ and assume the hypothesis of the lemma are satisfied. By Lemma 2.6, we have

$$
u(t) \leq \Gamma(q) \int_{0}^{1} \widetilde{g}(s) u(s) d s t^{q-1} E_{q, q}\left(-\widetilde{M} t^{q}\right)
$$

which implies that

$$
\int_{0}^{1} \widetilde{g}(s) u(s) d s \leq \Gamma(q) \int_{0}^{1} \widetilde{g}(s) u(s) d s \int_{0}^{1} s^{q-1} E_{q, q}\left(-\widetilde{M} s^{q}\right) \widetilde{g}(s) d s .
$$


Then, we have

$$
\int_{0}^{1} \widetilde{g}(s) u(s) d s\left(1-\Gamma(q) \int_{0}^{1} s^{q-1} E_{q, q}\left(-\widetilde{M} s^{q}\right) \widetilde{g}(s) d s\right) \leq 0 .
$$

Since $\Gamma(q) \int_{0}^{1} s^{q-1} E_{q, q}\left(-\widetilde{M} s^{q}\right) \widetilde{g}(s) d s<1$, we obtain $\int_{0}^{1} \widetilde{g}(s) u(s) d s \leq 0$. Using (2.4), one finds $t^{1-q} u(t) \leq$ 0 for all $t \in[0,1]$.

\section{EXISTENCE OF MINIMAL AND MAXIMAL SOLUTIONS FOR SYSTEMS WITH QUASIMONOTONE} INCREASING FUNCTIONS

In this section, we study the existence of minimal and maximal solutions of system (1.1) when the nonlinearities $f$ and $g$ are quasimonotone increasing.

On the nonlinearities $f$ and $g$, we impose the following conditions

(H1) $f:(0,1] \times \mathbb{R}^{2} \rightarrow \mathbb{R}$ is a continuous function and there exists $M_{1}>0$ such that the function $u \longmapsto f(t, u, v)+M_{1} u$ is increasing for all $t \in(0,1]$ and $v \in \mathbb{R}$.

(H2) $g:(0,1] \times \mathbb{R}^{2} \rightarrow \mathbb{R}$ is a continuous function and there exists $M_{2}>0$ such that the function $v \longmapsto g(t, u, v)+M_{2} v$ is increasing for all $t \in(0,1]$ and $u \in \mathbb{R}$.

(H3) $f(t, u, v)$ is increasing in $v$ for all fixed $t \in(0,1]$, and $u \in \mathbb{R}$.

(H4) $g(t, u, v)$ is increasing in $u$ for all fixed $t \in(0,1]$, and $v \in \mathbb{R}$.

(H5) The function $t \mapsto t^{1-\alpha_{1}} f(t, u(t), v(t))$ is a continuous function in $[0,1]$ for all $u \in C_{1-\alpha_{1}}([0,1])$ and $v \in C_{1-\alpha_{2}}([0,1])$.

(H6) The function $t \mapsto t^{1-\alpha_{2}} g(t, u(t), v(t))$ is a continuous function in $[0,1]$ for all $u \in C_{1-\alpha_{1}}([0,1])$ and $v \in C_{1-\alpha_{2}}([0,1])$.

Definition 3.1. We say that $(u, v)$ is a solution of (1.1) if

(i) $(u, v) \in C_{1-\alpha_{1}}([0,1]) \times C_{1-\alpha_{2}}([0,1])$,

(ii) $(u, v)$ satisfies $(1.1)$.

Definition 3.2. We say that $(\underline{u}, \underline{v}),(\bar{u}, \bar{v})$ is a pair of lower-upper solutions of type 1 for problem (1.1) if

(i) $(\underline{u}, \bar{u}) \in\left(C_{1-\alpha_{1}}([0,1])\right)^{2}$ and $(\underline{v}, \bar{v}) \in\left(C_{1-\alpha_{2}}([0,1])\right)^{2}$,

(ii) $\left\{\begin{array}{l}D^{\alpha_{1}} \underline{u}(t) \leq f(t, \underline{u}, \underline{v}), t \in(0,1], \\ D^{\alpha_{1}} \bar{u}(t) \geq f(t, \bar{u}, \bar{v}), t \in(0,1], \\ D^{\alpha_{2}} \underline{v}(t) \leq g(t, \underline{u}, \underline{v}), t \in(0,1], \\ D^{\alpha_{2}} \bar{v}(t) \geq g(t, \bar{u}, \bar{v}), t \in(0,1],\end{array}\right.$

(iii) $\left\{\begin{array}{l}\left.t^{1-\alpha_{1}} \underline{u}(t)\right|_{t=0} \leq \int_{0}^{1} g_{1}(s) \underline{u}(s) d s, \\ \left.t^{1-\alpha_{1}} \bar{u}(t)\right|_{t=0} \geq \int_{0}^{1} g_{1}(s) \bar{u}(s) d s, \\ \left.t^{1-\alpha_{2}} \underline{v}(t)\right|_{t=0} \leq \int_{0}^{1} g_{2}(s) \underline{v}(s) d s \\ \left.t^{1-\alpha_{2}} \bar{v}(t)\right|_{t=0} \geq \int_{0}^{1} g_{2}(s) \bar{v}(s) d s .\end{array}\right.$

Theorem 3.3. Assume that the hypothesis (Hi) for $i=1, \ldots, 6$ are satisfied and let $(\underline{u}, \underline{v}),(\bar{u}, \bar{v})$ be a pair of lower-upper solutions of type 1 for problem (1.1) such that $t^{1-\alpha_{1}} \underline{u}(t) \leq t^{1-\alpha_{1}} \bar{u}(t)$ and $t^{1-\alpha_{2}} \underline{v}(t) \leq$ $t^{1-\alpha_{2}} \bar{v}(t), \forall t \in[0,1]$. Then problem (1.1) has a minimal solution $\left(u_{*}, v_{*}\right)$ and a maximal solution $\left(u^{*}, v^{*}\right)$ such that for every solution $(u, v)$ of $(1.1)$ with $t^{1-\alpha_{1}} \underline{u}(t) \leq t^{1-\alpha_{1}} u(t) \leq t^{1-\alpha_{1}} \bar{u}(t)$ and $t^{1-\alpha_{2}} \underline{v}(t) \leq$ $t^{1-\alpha_{2}} v(t) \leq t^{1-\alpha_{2}} \bar{v}(t), \forall t \in[0,1]$, we have

$$
t^{1-\alpha_{1}} \underline{u}(t) \leq t^{1-\alpha_{1}} u_{*}(t) \leq t^{1-\alpha_{1}} u(t) \leq t^{1-\alpha_{1}} u^{*}(t) \leq t^{1-\alpha_{1}} \bar{u}(t) \text { in }[0,1]
$$


and

$$
t^{1-\alpha_{2}} \underline{v}(t) \leq t^{1-\alpha_{2}} v_{*}(t) \leq t^{1-\alpha_{2}} v(t) \leq t^{1-\alpha_{2}} v^{*}(t) \leq t^{1-\alpha_{2}} \bar{v}(t) \text { in }[0,1]
$$

Proof. The proof will be given in several steps.

We take $\underline{u}_{0}=\underline{u}, \underline{v}_{0}=\underline{v}$ and we define the sequences of functions $\left\{\underline{u}_{n}\right\}_{n \geq 1},\left\{\underline{v}_{n}\right\}_{n \geq 1}$ by

$$
\begin{aligned}
& \left\{\begin{array}{l}
D^{\alpha_{1}} \underline{u}_{n+1}(t)+M_{1} \underline{u}_{n+1}(t)=\widetilde{f}_{n}(t), t \in(0,1], \\
\left.t^{1-\alpha_{1}} \underline{u}_{n+1}(t)\right|_{t=0}=\int_{0}^{1} g_{1}(s) \underline{u}_{n}(s) d s,
\end{array}\right. \\
& \left\{\begin{array}{l}
D^{\alpha_{2}} \underline{v}_{n+1}(t)+M_{2} \underline{v}_{n+1}(t)=\widetilde{g}_{n}(t), t \in(0,1], \\
\left.t^{1-\alpha_{2}} \underline{v}_{n+1}(t)\right|_{t=0}=\int_{0}^{1} g_{2}(s) \underline{v}_{n}(s) d s,
\end{array}\right.
\end{aligned}
$$

where

$$
\widetilde{f}_{n}(t):=f\left(t, \underline{u}_{n}(t), \underline{v}_{n}(t)\right)+M_{1} \underline{u}_{n}(t),
$$

and

$$
\widetilde{g}_{n}(t):=g\left(t, \underline{u}_{n}(t), \underline{v}_{n}(t)\right)+M_{2} \underline{v}_{n}(t) .
$$

Analogously, we take $\bar{u}_{0}=\bar{u}, \bar{v}_{0}=\bar{v}$ and we define the sequences of functions $\left\{\bar{u}_{n}\right\}_{n \geq 1},\left\{\bar{v}_{n}\right\}_{n \geq 1}$ by

$$
\begin{aligned}
& \left\{\begin{array}{l}
D^{\alpha_{1}} \bar{u}_{n+1}(t)+M_{1} \bar{u}_{n+1}(t)=\widehat{f}_{n}(t), t \in(0,1], \\
\left.t^{1-\alpha_{1}} \bar{u}_{n+1}(t)\right|_{t=0}=\int_{0}^{1} g_{1}(s) \bar{u}_{n}(s) d s,
\end{array}\right. \\
& \left\{\begin{array}{l}
D^{\alpha_{2}} \bar{v}_{n+1}(t)+M_{2} \bar{v}_{n+1}(t)=\widehat{g}_{n}(t), t \in(0,1], \\
\left.t^{1-\alpha_{2}} \bar{v}_{n+1}(t)\right|_{t=0}=\int_{0}^{1} g_{2}(s) \bar{v}_{n}(s) d s,
\end{array}\right.
\end{aligned}
$$

where

$$
\widehat{f}_{n}(t):=f\left(t, \bar{u}_{n}(t), \bar{v}_{n}(t)\right)+M_{1} \bar{u}_{n}(t),
$$

and

$$
\widehat{g}_{n}(t):=g\left(t, \bar{u}_{n}(t), \bar{v}_{n}(t)\right)+M_{2} \bar{v}_{n}(t) .
$$

Step 1. The sequences of functions $\left\{\left(\underline{u}_{n}, \underline{v}_{n}\right)\right\}_{n \in \mathbb{N}}$ is well defined. For all $n \in \mathbb{N}$, we have

$$
\left(\underline{u}_{n}, \underline{v}_{n}\right) \in C_{1-\alpha_{1}}([0,1]) \times C_{1-\alpha_{2}}([0,1]) .
$$

For $n=0$, we have $\left(\underline{u}_{0}, \underline{v}_{0}\right)=(\underline{u}, \underline{v})$ and $(\underline{u},, \underline{v}) \in C_{1-\alpha_{1}}([0,1]) \times C_{1-\alpha_{2}}([0,1])$. Assume that for fixed $n \geq 1,\left(\underline{u}_{n}, \underline{v}_{n}\right)$ is well defined. It follows that

$$
\left(\underline{u}_{n}, \underline{v}_{n}\right) \in C_{1-\alpha_{1}}([0,1]) \times C_{1-\alpha_{2}}([0,1]) .
$$

We show that $\left(\underline{u}_{n+1}, \underline{v}_{n+1}\right)$ is well defined and we have

$$
\left(\underline{u}_{n+1}, \underline{v}_{n+1}\right) \in C_{1-\alpha_{1}}([0,1]) \times C_{1-\alpha_{2}}([0,1]) .
$$

By (3.1) and (3.2), we have

$$
\begin{aligned}
& \left\{\begin{array}{l}
D^{\alpha_{1}} \underline{u}_{n+1}(t)+M_{1} \underline{u}_{n+1}(t)=\widetilde{f}_{n}(t), t \in(0,1], \\
\left.t^{1-\alpha_{1}} \underline{u}_{n+1}(t)\right|_{t=0}=\int_{0}^{1} g_{1}(s) \underline{u}_{n}(s) d s,
\end{array}\right. \\
& \left\{\begin{array}{l}
D^{\alpha_{2}} \underline{v}_{n+1}(t)+M_{2} \underline{v}_{n+1}(t)=\widetilde{f}_{n}(t), t \in(0,1], \\
\left.t^{1-\alpha_{2}} \underline{v}_{n+1}(t)\right|_{t=0}=\int_{0}^{1} g_{2}(s) \underline{v}_{n}(s) d s
\end{array}\right.
\end{aligned}
$$


Since $\left(\underline{u}_{n}, \underline{v}_{n}\right) \in C_{1-\alpha_{1}}([0,1]) \times C_{1-\alpha_{2}}([0,1])$ and using hypothesis (H1), (H2), (H5) and (H6), we obtain that the functions $F_{n} \in C([0,1])$ and $G_{n} \in C([0,1])$, where

$$
F_{n}(t):=t^{1-\alpha_{1}} \widetilde{f}_{n}(t),
$$

and

$$
G_{n}(t):=t^{1-\alpha_{2}} \widetilde{g}_{n}(t) .
$$

Then by Lemma 2.6 , we find that $\left(\underline{u}_{n+1}, \underline{v}_{n+1}\right)$ is well defined. Hence,

$$
\left(\underline{u}_{n+1}, \underline{v}_{n+1}\right) \in C_{1-\alpha_{1}}([0,1]) \times C_{1-\alpha_{2}}([0,1]) .
$$

For all $n \in \mathbb{N}$, the sequence of functions $\left\{\left(\underline{u}_{n}, \underline{v}_{n}\right)\right\}_{n \in \mathbb{N}}$ is well defined. So

$$
\left(\underline{u}_{n}, \underline{v}_{n}\right) \in C_{1-\alpha_{1}}([0,1]) \times C_{1-\alpha_{2}}([0,1]) .
$$

Step 2. The sequence of functions $\left\{\left(\bar{u}_{n}, \bar{v}_{n}\right)\right\}_{n \in \mathbb{N}}$ is well defined. For all $n \in \mathbb{N}$, we have

$$
\left(\bar{u}_{n}, \bar{v}_{n}\right) \in C_{1-\alpha_{1}}([0,1]) \times C_{1-\alpha_{2}}([0,1]) .
$$

The proof is similar to that of Step 1, so it is omitted here.

Step 3. For all $n \in \mathbb{N}$, we have

$$
t^{1-\alpha_{1}} \underline{u}_{n}(t) \leq t^{1-\alpha_{1}} \underline{u}_{n+1}(t) \leq t^{1-\alpha_{1}} \bar{u}_{n+1}(t) \leq t^{1-\alpha_{1}} \bar{u}_{n}(t) \text { in }[0,1],
$$

and

$$
t^{1-\alpha_{2}} \underline{v}_{n}(t) \leq t^{1-\alpha_{2}} \underline{v}_{n+1}(t) \leq t^{1-\alpha_{2}} \bar{v}_{n+1}(t) \leq t^{1-\alpha_{2}} \bar{v}_{n}(t) \text { in }[0,1]
$$

Let

$$
\begin{gathered}
w_{0}(t):=\underline{u}_{1}(t)-\underline{u}_{0}(t) \text { and } z_{0}(t):=\underline{v}_{1}(t)-\underline{v}_{0}(t), t \in(0,1], \\
\left.t^{1-\alpha_{1}} w_{0}(t)\right|_{t=0}=\left.t^{1-\alpha_{1}} \underline{u}_{1}(t)\right|_{t=0}-\left.t^{1-\alpha_{1}} \underline{u}_{0}(t)\right|_{t=0},
\end{gathered}
$$

and

$$
\left.t^{1-\alpha_{2}} z_{0}(t)\right|_{t=0}=\left.t^{1-\alpha_{2}} \underline{v}_{1}(t)\right|_{t=0}-\left.t^{1-\alpha_{2}} \underline{v}_{0}(t)\right|_{t=0} .
$$

By (3.1), (3.2) and using the Definition 3.2, we have

$$
\left\{\begin{array}{l}
D^{\alpha_{1}} w_{0}(t)+M_{1} w_{0}(t) \geq 0, t \in(0,1], \\
\left.t^{1-\alpha_{1}} w_{0}(t)\right|_{t=0} \geq 0
\end{array}\right.
$$

and

$$
\left\{\begin{array}{l}
D_{0}^{\alpha_{2}} z_{0}(t)+M_{1} z_{0}(t) \geq 0, t \in(0,1] \\
\left.t^{1-\alpha_{2}} z_{0}(t)\right|_{t=0} \geq 0
\end{array}\right.
$$

Using Lemma 2.10, we obtain

$$
t^{1-\alpha_{1}} w_{0}(t) \geq 0 \text { and } t^{1-\alpha_{2}} z_{0}(t) \geq 0 \text { in }[0,1],
$$

that is,

$$
t^{1-\alpha_{1}} \underline{u}_{0}(t) \leq t^{1-\alpha_{1}} \underline{u}_{1}(t) \text { in }[0,1]
$$

and

$$
t^{1-\alpha_{2}} \underline{v}_{0}(t) \leq t^{1-\alpha_{2}} \underline{v}_{1}(t) \text { in }[0,1]
$$

Similarly, we can prove that

$$
t^{1-\alpha_{1}} \bar{u}_{1}(t) \leq t^{1-\alpha_{1}} \bar{u}_{0}(t) \text { in }[0,1]
$$


and

$$
t^{1-\alpha_{2}} \bar{v}_{1}(t) \leq t^{1-\alpha_{2}} \bar{v}_{0}(t) \text { in }[0,1]
$$

Now, we put by definition

$$
\begin{gathered}
p_{1}(t)=\underline{u}_{1}(t)-\bar{u}_{1}(t) \text { and } y_{1}(t)=\underline{v}_{1}(t)-\bar{v}_{1}(t), t \in(0,1], \\
\left.t^{1-\alpha_{1}} p_{1}(t)\right|_{t=0}=\left.t^{1-\alpha_{1}} \underline{u}_{1}(t)\right|_{t=0}-\left.t^{1-\alpha_{1}} \bar{u}_{1}(t)\right|_{t=0},
\end{gathered}
$$

and

$$
\left.t^{1-\alpha_{2}} y_{1}(t)\right|_{t=0}=\left.t^{1-\alpha_{2}} \underline{v}_{1}(t)\right|_{t=0}-\left.t^{1-\alpha_{2}} \bar{v}_{1}(t)\right|_{t=0} .
$$

By (3.1), (3.2), (3.3) and (3.4), we have

$$
\left\{\begin{array}{l}
D^{\alpha_{1}} p_{1}(t)+M_{1} p_{1}(t)=\widetilde{f}_{0}(t)-\widehat{f}_{0}(t), t \in(0,1], \\
\left.t^{1-\alpha_{1}} p_{1}(t)\right|_{t=0}=\int_{0}^{1} g_{1}(s)\left(\underline{u}_{0}(s)-\bar{u}_{0}(s)\right) d s
\end{array}\right.
$$

and

Since

$$
\left\{\begin{array}{l}
D^{\alpha_{2}} y_{1}(t)+M_{2} y_{1}(t)=\widetilde{g}_{0}(t)-\widehat{g}_{0}(t), t \in(0,1] \\
\left.t^{1-\alpha_{2}} y_{1}(t)\right|_{t=0}=\int_{0}^{1} g_{2}(s)\left(\underline{v}_{0}(s)-\bar{v}_{0}(s)\right) d s
\end{array}\right.
$$

$$
t^{1-\alpha_{1}} \underline{u}_{0}(t)=t^{1-\alpha_{1}} \underline{u}(t) \leq t^{1-\alpha_{1}} \bar{u}(t)=t^{1-\alpha_{1}} \bar{u}_{0}(t) \text { in }[0,1],
$$

and

$$
t^{1-\alpha_{2}} \underline{v}_{0}(t)=t^{1-\alpha_{2}} \underline{v}(t) \leq t^{1-\alpha_{2}} \bar{v}(t)=t^{1-\alpha_{2}} \bar{v}_{0}(t) \text { in }[0,1],
$$

and using the hypothesis $(\mathrm{Hi})$ for $\mathrm{i}=1, \ldots, 4$, we obtain

$$
\left\{\begin{array}{l}
D^{\alpha_{1}} p_{1}(t)+M_{1} p_{1}(t) \leq 0, t \in(0,1], \\
\left.t^{1-\alpha_{1}} p_{1}(t)\right|_{t=0}=\int_{0}^{1} g_{1}(s) s^{\alpha_{1}-1} s^{1-\alpha_{1}}\left(\underline{u}_{0}(s)-\bar{u}_{0}(s)\right) d s \leq 0,
\end{array}\right.
$$

and

$$
\left\{\begin{array}{l}
D^{\alpha_{2}} y_{1}(t)+M_{2} y_{1}(t) \leq 0, t \in(0,1], \\
\left.t^{1-\alpha_{1}} y_{1}(t)\right|_{t=0}=\int_{0}^{1} g_{2}(s) s^{\alpha_{2}-1} s^{1-\alpha_{2}}\left(\underline{v}_{0}(s)-\bar{v}_{0}(s)\right) d s \leq 0 .
\end{array}\right.
$$

Using Lemma 2.10, we obtain

$$
t^{1-\alpha_{1}} p_{1}(t) \leq 0 \text { and } t^{1-\alpha_{2}} y_{1}(t) \leq 0 \text { in }[0,1],
$$

that is,

$$
t^{1-\alpha_{1}} \underline{u}_{1}(t) \leq t^{1-\alpha_{1}} \bar{u}_{1}(t) \text { in }[0,1]
$$

and

$$
t^{1-\alpha_{2}} \underline{v}_{1}(t) \leq t^{1-\alpha_{2}} \bar{v}_{1}(t) \text { in }[0,1] .
$$

In view of (3.5), (3.6), (3.7), (3.8), (3.9) and (3.10), we have

$$
t^{1-\alpha_{1}} \underline{u}_{0}(t) \leq t^{1-\alpha_{1}} \underline{u}_{1}(t) \leq t^{1-\alpha_{1}} \bar{u}_{1}(t) \leq t^{1-\alpha_{1}} \bar{u}_{0}(t) \text { in }[0,1],
$$

and

$$
t^{1-\alpha_{2}} \underline{v}_{0}(t) \leq t^{1-\alpha_{2}} \underline{v}_{1}(t) \leq t^{1-\alpha_{2}} \bar{v}_{1}(t) \leq t^{1-\alpha_{2}} \bar{v}_{0}(t) \text { in }[0,1] .
$$

Assume, for fixed $n \geq 1$, that

$$
t^{1-\alpha_{1}} \underline{u}_{n}(t) \leq t^{1-\alpha_{1}} \underline{u}_{n+1}(t) \leq t^{1-\alpha_{1}} \bar{u}_{n+1}(t) \leq t^{1-\alpha_{1}} \bar{u}_{n}(t) \text { in }[0,1],
$$

and

$$
t^{1-\alpha_{2}} \underline{v}_{n}(t) \leq t^{1-\alpha_{2}} \underline{v}_{n+1}(t) \leq t^{1-\alpha_{2}} \bar{v}_{n+1}(t) \leq t^{1-\alpha_{2}} \bar{v}_{n}(t) \text { in }[0,1]
$$


We show that

$$
t^{1-\alpha_{1}} \underline{u}_{n+1}(t) \leq t^{1-\alpha_{1}} \underline{u}_{n+2}(t) \leq t^{1-\alpha_{1}} \bar{u}_{n+2}(t) \leq t^{1-\alpha_{1}} \bar{u}_{n+1}(t) \text { in }[0,1]
$$

and

$$
t^{1-\alpha_{2}} \underline{v}_{n+1}(t) \leq t^{1-\alpha_{2}} \underline{v}_{n+2}(t) \leq t^{1-\alpha_{2}} \bar{v}_{n+2}(t) \leq t^{1-\alpha_{2}} \bar{v}_{n+1}(t) \text { in }[0,1] .
$$

We put by definition

$$
\begin{gathered}
w_{n+1}(t):=\underline{u}_{n+2}(t)-\underline{u}_{n+1}(t) \text { and } z_{n+1}(t):=\underline{v}_{n+2}(t)-\underline{v}_{n+1}(t), t \in(0,1], \\
\left.t^{1-\alpha_{1}} w_{n+1}(t)\right|_{t=0}=\left.t^{1-\alpha_{1}} \underline{u}_{n+2}(t)\right|_{t=0}-\left.t^{1-\alpha_{1}} \underline{u}_{n+1}(t)\right|_{t=0},
\end{gathered}
$$

and

$$
\left.t^{1-\alpha_{2}} z_{n+1}(t)\right|_{t=0}=\left.t^{1-\alpha_{2}} \underline{v}_{n+2}(t)\right|_{t=0}-\left.t^{1-\alpha_{2}} \underline{v}_{n+1}(t)\right|_{t=0} .
$$

By (3.1) and (3.2), we have

$$
\left\{\begin{array}{l}
D^{\alpha_{1}} w_{n+1}(t)+M_{1} w_{n+1}(t)=\widetilde{f}_{n+1}(t)-\widetilde{f}_{n}(t), t \in(0,1], \\
\left.t^{1-\alpha_{1}} w_{n+1}(t)\right|_{t=0}=\int_{0}^{1} g_{1}(s)\left(\underline{u}_{n+1}(s)-\underline{u}_{n}(s)\right) d s
\end{array}\right.
$$

and

$$
\left\{\begin{array}{l}
D^{\alpha_{2}} z_{n+1}(t)+M_{2} z_{n+1}(t)=\widetilde{g}_{n+1}(t)-\widetilde{g}_{n}(t), t \in(0,1], \\
\left.t^{1-\alpha_{2}} z_{n+1}(t)\right|_{t=0}=\int_{0}^{1} g_{2}(s)\left(\underline{v}_{n+1}(s)-\underline{v}_{n+1}(s)\right) d s .
\end{array}\right.
$$

Since by the hypothesis of recurrence, we have $t^{1-\alpha_{1}} \underline{u}_{n}(t) \leq t^{1-\alpha_{1}} \underline{u}_{n+1}(t)$ and $t^{1-\alpha_{2}} \underline{v}_{n}(t) \leq t^{1-\alpha_{2}} \underline{v}_{n+1}(t)$ in $[0,1]$. Using the hypothesis $(\mathrm{Hi})$ for $i=1, \ldots, 4$, we obtain

$$
\left\{\begin{array}{l}
D^{\alpha_{1}} w_{n+1}(t)+M_{1} w_{n+1}(t) \geq 0, t \in(0,1] \\
\left.t^{1-\alpha_{1}} w_{n+1}(t)\right|_{t=0} \geq 0
\end{array}\right.
$$

and

Using Lemma 2.10, we obtain

$$
\left\{\begin{array}{l}
D^{\alpha_{2}} z_{n+1}(t)+M_{2} z_{n+1}(t) \geq 0, t \in(0,1] \\
\left.t^{1-\alpha_{2}} z_{n+1}(t)\right|_{t=0} \geq 0
\end{array}\right.
$$

$$
t^{1-\alpha_{1}} w_{n+1}(t) \geq 0 \text { and } t^{1-\alpha_{2}} z_{n+1}(t) \geq 0 \text { in }[0,1]
$$

that is,

$$
t^{1-\alpha_{1}} \underline{u}_{n+1}(t) \leq t^{1-\alpha_{1}} \underline{u}_{n+2}(t) \text { and } t^{1-\alpha_{2}} \underline{v}_{n+1}(t) \leq t^{1-\alpha_{2}} \underline{v}_{n+2}(t) \text { in }[0,1] .
$$

Similarly, we can prove that

$$
t^{1-\alpha_{1}} \bar{u}_{n+2}(t) \leq t^{1-\alpha_{1}} \bar{u}_{n+1}(t) \text { and } t^{1-\alpha_{2}} \bar{v}_{n+2}(t) \leq t^{1-\alpha_{2}} \bar{v}_{n+1}(t) \text { in }[0,1],
$$

and

$$
t^{1-\alpha_{1}} \underline{u}_{n+2}(t) \leq t^{1-\alpha_{1}} \bar{u}_{n+2}(t) \text { and } t^{1-\alpha_{2}} \underline{v}_{n+2}(t) \leq t^{1-\alpha_{2}} \bar{v}_{n+2}(t) \text { in }[0,1] .
$$

In view of (3.11), (3.12) and (3.13), we have

$$
t^{1-\alpha_{1}} \underline{u}_{n+1}(t) \leq t^{1-\alpha_{1}} \underline{u}_{n+2}(t) \leq t^{1-\alpha_{1}} \bar{u}_{n+2}(t) \leq t^{1-\alpha_{1}} \bar{u}_{n+1}(t) \text { in }[0,1],
$$

and

$$
t^{1-\alpha_{2}} \underline{v}_{n+1}(t) \leq t^{1-\alpha_{2}} \underline{v}_{n+2}(t) \leq t^{1-\alpha_{2}} \bar{v}_{n+2}(t) \leq t^{1-\alpha_{2}} \bar{v}_{n+1}(t) \text { in }[0,1] .
$$

Hence for all $n \in \mathbb{N}$, we have

$$
t^{1-\alpha_{1}} \underline{u}_{n}(t) \leq t^{1-\alpha_{1}} \underline{u}_{n+1}(t) \leq t^{1-\alpha_{1}} \bar{u}_{n+1}(t) \leq t^{1-\alpha_{1}} \bar{u}_{n}(t) \text { in }[0,1],
$$


and

$$
t^{1-\alpha_{2}} \underline{v}_{n}(t) \leq t^{1-\alpha_{2}} \underline{v}_{n+1}(t) \leq t^{1-\alpha_{2}} \bar{v}_{n+1}(t) \leq t^{1-\alpha_{2}} \bar{v}_{n}(t) \text { in }[0,1]
$$

The proof of Step 3 is complete.

Step 4. The sequences of functions $\left\{t^{1-\alpha_{1}} \underline{u}_{n}\right\}_{n \in \mathbb{N}},\left\{\left(t^{1-\alpha_{2}} \underline{v}_{n}\right\}_{n \in \mathbb{N}}\right.$ are uniformly bounded on $[0,1]$. Letting $n \in \mathbb{N}$ and using Step 3, we have

$$
t^{1-\alpha_{1}} \underline{u}(t) \leq t^{1-\alpha_{1}} \underline{u}_{n}(t) \leq t^{1-\alpha_{1}} \bar{u}(t) \text { in }[0,1] .
$$

Since the functions $t \longmapsto t^{1-\alpha_{1}} \underline{u}(t)$ and $t \longmapsto t^{1-\alpha_{1}} \bar{u}(t)$ are continuous on $[0,1]$, we find that they are bounded on $[0,1]$. Hence, the sequence of functions $\left\{t^{1-\alpha_{1}} \underline{u}_{n}\right\}_{n \in \mathbb{N}}$ is uniformly bounded on $[0,1]$. Similarly, we can prove that the sequences of functions $\left\{\left(t^{1-\alpha_{2}} \underline{v}_{n}\right\}_{n \in \mathbb{N}}\right.$ is uniformly bounded on $[0,1]$.

Step 5. The sequences of functions $\left\{t^{1-\alpha_{1}} \bar{u}_{n}\right\}_{n \in \mathbb{N}}$ and $\left\{t^{1-\alpha_{2}} \bar{v}_{n}\right\}_{n \in \mathbb{N}}$ are uniformly bounded on $[0,1]$.

The proof is similar to that of Step 4, so it is omitted.

Now we are in a position to prove that the sequences of functions $\left\{t^{1-\alpha_{1}} \underline{u}_{n}\right\}_{n \in \mathbb{N}}$ and $\left\{t^{1-\alpha_{2}} \underline{v}_{n}\right\}_{n \in \mathbb{N}}$ are equicontinuous on $[0,1]$. The idea of the proof is similar to that used in [19, Page 5].

Step 6. The sequences of functions $\left\{t^{1-\alpha_{1}} \underline{u}_{n}\right\}_{n \in \mathbb{N}}$ and $\left\{t^{1-\alpha_{2}} \underline{v}_{n}\right\}_{n \in \mathbb{N}}$ are equicontinuous on $[0,1]$.

Let $\varepsilon>0, t_{1}, t_{2} \in[0,1]$ such that $t_{1}<t_{2}$ and $n \in \mathbb{N}$. First, we have

$$
\lim _{t \rightarrow 0} t^{1-\alpha_{1}} \int_{0}^{t}(t-s)^{\alpha_{1}-1} s^{\alpha_{1}-1} d s=\lim _{t \rightarrow 0} \alpha^{\alpha_{1}} \beta\left(\alpha_{1}, \alpha_{1}\right)=0,
$$

where $\beta$ is the Beta Euler function defined by

$$
\beta\left(z_{1}, z_{2}\right)=\int_{0}^{1}(1-s)^{z_{1}-1} s^{z_{2}-1} d s,
$$

where $z_{1} \in \mathbb{C}, z_{2} \in \mathbb{C}$ with $\mathfrak{R}\left(z_{1}\right)>0$ and $\mathfrak{R}\left(z_{2}\right)>0$. Then there exists $\delta_{1}>0$ such that for $0<t<\delta_{1}$, we have

$$
t^{1-\alpha_{1}} \int_{0}^{t}(t-s)^{\alpha_{1}-1} s^{\alpha_{1}-1} d s<\varepsilon
$$

We distinguish two cases

Case 1. $0 \leq t_{1}<t_{2}<\delta_{1}$. By (3.1) and using Theorem 3.10 in [4], we have

$$
\begin{aligned}
& t_{2}^{1-\alpha_{1}} \underline{u}_{n+1}\left(t_{2}\right)-t_{1}^{1-\alpha_{1}} \underline{u}_{n+1}\left(t_{1}\right) \\
& =\frac{t_{2}^{1-\alpha_{1}}}{\Gamma\left(\alpha_{1}\right)} \int_{0}^{t_{2}}\left(t_{2}-s\right)^{\alpha_{1}-1} \widetilde{F}_{n}(s) d s-\frac{t_{1}^{1-\alpha_{1}}}{\Gamma\left(\alpha_{1}\right)} \int_{0}^{t_{1}}\left(t_{1}-s\right)^{\alpha_{1}-1} \widetilde{F}_{n}(s) d s,
\end{aligned}
$$

where

$$
\widetilde{F}_{n}(s):=f\left(s, \underline{u}_{n}(s), \underline{v}_{n}(s)\right)+M_{1}\left(\underline{u}_{n}(s)-\underline{u}_{n+1}(s)\right) .
$$

It follows that

$$
\begin{aligned}
& \left|t_{2}^{1-\alpha_{1}} \underline{u}_{n+1}\left(t_{2}\right)-t_{1}^{1-\alpha_{1}} \underline{u}_{n+1}\left(t_{1}\right)\right| \\
& \leq \frac{t_{2}^{1-\alpha_{1}}}{\Gamma\left(\alpha_{1}\right)} \int_{0}^{t_{2}}\left(t_{2}-s\right)^{\alpha_{1}-1}\left|\widetilde{F}_{n}(s)\right| d s+\frac{t_{1}^{1-\alpha_{1}}}{\Gamma\left(\alpha_{1}\right)} \int_{0}^{t_{1}}\left(t_{1}-s\right)^{\alpha_{1}-1}\left|\widetilde{F}_{n}(s)\right| d s,
\end{aligned}
$$

In view of Step 4 and hypothesis (H5), we have

$$
\exists \widehat{M}>0, \forall n \in \mathbb{N}, \forall s \in[0,1],\left|s^{1-\alpha_{1}} \widetilde{F}_{n}(s)\right| \leq \widehat{M},
$$


which implies that

$$
\begin{aligned}
& \left|t_{2}^{1-\alpha_{1}} \underline{u}_{n+1}\left(t_{2}\right)-t_{1}^{1-\alpha_{1}} \underline{u}_{n+1}\left(t_{1}\right)\right| \\
& \leq \frac{\widetilde{M}}{\Gamma\left(\alpha_{1}\right)}\left(t_{2}^{1-\alpha_{1}} \int_{0}^{t_{2}}\left(t_{2}-s\right)^{\alpha_{1}-1} s^{\alpha_{1}-1} d s+t_{1}^{1-\alpha_{1}} \int_{0}^{t_{1}}\left(t_{1}-s\right)^{\alpha_{1}-1} s^{\alpha_{1}-1} d s\right) \\
& \leq \frac{2 \widetilde{M}}{\Gamma\left(\alpha_{1}\right)} \varepsilon .
\end{aligned}
$$

Case 2. $\delta_{1} \leq t_{1}<t_{2} \leq 1$. In this case, we have

$$
\begin{aligned}
& t_{2}^{1-\alpha_{1}} \underline{u}_{n+1}\left(t_{2}\right)-t_{1}^{1-\alpha_{1}} \underline{u}_{n+1}\left(t_{1}\right) \\
& =\frac{1}{\Gamma\left(\alpha_{1}\right)} \int_{0}^{t_{1}}\left(t_{2}^{1-\alpha_{1}}\left(t_{2}-s\right)^{\alpha_{1}-1}-t_{1}^{1-\alpha_{1}}\left(t_{1}-s\right)^{\alpha_{1}-1}\right) \widetilde{F}_{n}(s) d s+\frac{t_{2}^{1-\alpha_{1}}}{\Gamma\left(\alpha_{1}\right)} \int_{t_{1}}^{t_{2}}\left(t_{2}-s\right)^{\alpha_{1}-1} \widetilde{F}_{n}(s) d s,
\end{aligned}
$$

which implies that

$$
\begin{aligned}
& \left|t_{2}^{1-\alpha_{1}} \underline{u}_{n+1}\left(t_{2}\right)-t_{1}^{1-\alpha_{1}} \underline{u}_{n+1}\left(t_{1}\right)\right| \leq \frac{\widehat{M}}{\Gamma\left(\alpha_{1}\right)} \int_{0}^{t_{1}}\left|t_{2}^{1-\alpha_{1}}\left(t_{2}-s\right)^{\alpha_{1}-1}-t_{1}^{1-\alpha_{1}}\left(t_{1}-s\right)^{\alpha_{1}-1}\right| s^{\alpha_{1}-1} d s \\
& +\frac{\widehat{M} t_{2}^{1-\alpha_{1}}}{\Gamma\left(\alpha_{1}\right)} \int_{t_{1}}^{t_{2}}\left(t_{2}-s\right)^{\alpha_{1}-1} s^{\alpha_{1}-1} d s \\
& \leq \frac{\widehat{M}}{\Gamma\left(\alpha_{1}\right)} \int_{0}^{t_{1}}\left|t_{2}^{1-\alpha_{1}}\left(t_{2}-s\right)^{\alpha_{1}-1}-t_{1}^{1-\alpha_{1}}\left(t_{1}-s\right)^{\alpha_{1}-1}\right| s^{\alpha_{1}-1} d s \\
& +\frac{\widehat{M} t_{2}^{1-\alpha_{1}}}{\Gamma\left(\alpha_{1}+1\right)} \delta_{1}^{\alpha_{1}-1}\left(t_{2}-t_{1}\right)^{\alpha_{1}} \\
& \leq \frac{\widehat{M}}{\Gamma\left(\alpha_{1}\right)} \int_{0}^{\delta_{1}}\left|t_{2}^{1-\alpha_{1}}\left(t_{2}-s\right)^{\alpha_{1}-1}-t_{1}^{1-\alpha_{1}}\left(t_{1}-s\right)^{\alpha_{1}-1}\right| s^{\alpha_{1}-1} d s \\
& +\frac{\widehat{M} \delta_{1}^{\alpha_{1}-1}}{\Gamma\left(\alpha_{1}\right)} \int_{\delta_{1}}^{t_{1}}\left(t_{1}^{1-\alpha_{1}}\left(t_{1}-s\right)^{\alpha_{1}-1}-t_{2}^{1-\alpha_{1}}\left(t_{2}-s\right)^{\alpha_{1}-1}\right) d s \\
& +\frac{\widehat{M} t_{2}^{1-\alpha_{1}}}{\Gamma\left(\alpha_{1}+1\right)} \delta_{1}^{\alpha_{1}-1}\left(t_{2}-t_{1}\right)^{\alpha_{1}} \\
& \leq \frac{\widehat{M}}{\Gamma\left(\alpha_{1}\right)} \int_{0}^{\delta_{1}}\left|t_{2}^{1-\alpha_{1}}\left(t_{2}-s\right)^{\alpha_{1}-1}-t_{1}^{1-\alpha_{1}}\left(t_{1}-s\right)^{\alpha_{1}-1}\right| s^{\alpha_{1}-1} d s \\
& +\frac{\widehat{M} \delta_{1}^{\alpha_{1}-1}}{\Gamma\left(\alpha_{1}+1\right)}\left(t_{1}^{1-\alpha_{1}}\left(t_{1}-\delta_{1}\right)^{\alpha_{1}}-t_{2}^{1-\alpha_{1}}\left(t_{2}-\delta_{1}\right)^{\alpha_{1}}\right) \\
& +2 \frac{\widehat{M} t_{2}^{1-\alpha_{1}}}{\Gamma\left(\alpha_{1}+1\right)} \delta_{1}^{\alpha_{1}-1}\left(t_{2}-t_{1}\right)^{\alpha_{1}}
\end{aligned}
$$

that is,

$$
\begin{aligned}
& \left|t_{2}^{1-\alpha_{1}} \underline{u}_{n+1}\left(t_{2}\right)-t_{1}^{1-\alpha_{1}} \underline{u}_{n+1}\left(t_{1}\right)\right| \\
& \leq \frac{\widehat{M}}{\Gamma\left(\alpha_{1}\right)} \int_{0}^{\delta_{1}}\left|t_{2}^{1-\alpha_{1}}\left(t_{2}-s\right)^{\alpha_{1}-1}-t_{1}^{1-\alpha_{1}}\left(t_{1}-s\right)^{\alpha_{1}-1}\right| s^{\alpha_{1}-1} d s \\
& +\frac{\widehat{M} \delta_{1}^{\alpha_{1}-1}}{\Gamma\left(\alpha_{1}+1\right)}\left(t_{1}^{1-\alpha_{1}}\left(t_{1}-\delta_{1}\right)^{\alpha_{1}}-t_{2}^{1-\alpha_{1}}\left(t_{2}-\delta_{1}\right)^{\alpha_{1}}\right)+2 \frac{\widehat{M} t_{2}^{1-\alpha_{1}}}{\Gamma\left(\alpha_{1}+1\right)} \delta_{1}^{\alpha_{1}-1}\left(t_{2}-t_{1}\right)^{\alpha_{1}} .
\end{aligned}
$$


Note that the functions $t \mapsto t^{1-\alpha_{1}}(t-s)^{\alpha_{1}-1}$ and $t \mapsto t^{\alpha}$ are continuous. If $t_{2}-t_{1}<\delta_{2}$, there exists $\delta_{2}>0$ such that

$$
\left|t_{2}^{1-\alpha_{1}}\left(t_{2}-s\right)^{\alpha_{1}-1}-t_{1}^{1-\alpha_{1}}\left(t_{1}-s\right)^{\alpha_{1}-1}\right|<\frac{\Gamma\left(\alpha_{1}+1\right)}{3 \widehat{M} \delta_{1}^{\alpha_{1}-1}} \varepsilon
$$

and

$$
\left(t_{2}-t_{1}\right)^{\alpha_{1}}<\frac{\Gamma\left(\alpha_{1}+1\right)}{6 \widehat{M} t_{2}^{1-\alpha_{1}} \delta_{1}^{\alpha_{1}-1}} \varepsilon
$$

If $t_{2}-t_{1}<\delta_{2}$, we obtain from (3.15) and (3.16) that

$$
\left|t_{2}^{1-\alpha_{1}} \underline{u}_{n+1}\left(t_{2}\right)-t_{1}^{1-\alpha_{1}} \underline{u}_{n+1}\left(t_{1}\right)\right|<\frac{\delta_{1}}{3} \varepsilon+\frac{\varepsilon}{3}+\frac{\varepsilon}{3}<\varepsilon .
$$

Therefore the sequence of functions $\left\{t^{1-\alpha_{1}} \underline{u}_{n}\right\}_{n \in \mathbb{N}}$ is equicontinuous on [0,1]. Similarly, we can prove that the sequence of functions $\left\{t^{1-\alpha_{2}} \underline{v}_{n}\right\}_{n \in \mathbb{N}}$ is equicontinuous on $[0,1]$.

Step 7. The sequences of functions $\left\{t^{1-\alpha_{1}} \bar{u}_{n}\right\}_{n \in \mathbb{N}}$ and $\left\{t^{1-\alpha_{2}} \bar{v}_{n}\right\}_{n \in \mathbb{N}}$ are equicontinuous on $[0,1]$.

The proof is similar to that of Step 6, so it is omitted.

Step 8. The consequence $\left\{\left(\underline{u}_{n}, \underline{v}_{n}\right)\right\}_{n \in \mathbb{N}}$ converges to a minimal solution of (1.1).

By Step 4 and Step 6, the sequence of functions $\left\{t^{1-\alpha_{1}} \underline{u}_{n}\right\}_{n \in \mathbb{N}}$ and $\left\{t^{1-\alpha_{2}} \underline{v}_{n}\right\}_{n \in \mathbb{N}}$ are uniformly bounded on $C([0,1])$ and equicontinuous on $[0,1]$. Using the Arzéla-Ascoli theorem, we see that there exists a subsequence $\left\{\left(t^{1-\alpha_{1}} \underline{u}_{n_{j}}, t^{1-\alpha_{2}} \underline{v}_{n_{j}}\right)\right\}_{n_{j} \in \mathbb{N}}$ of $\left\{\left(t^{1-\alpha_{1}} \underline{u}_{n}, t^{1-\alpha_{2}} \underline{v}_{n}\right)\right\}_{n \in \mathbb{N}}$, which converges in $C([0,1]) \times$ $C([0,1])$. Let

$$
\left(t^{1-\alpha_{1}} u, t^{1-\alpha_{2}} v\right):=\lim _{n_{j} \rightarrow+\infty}\left(t^{1-\alpha_{1}} \underline{u}_{n_{j}}, t^{1-\alpha_{2}} \underline{v}_{n_{j}}\right) .
$$

From Step 3, the sequences $\left\{t^{1-\alpha_{1}} \underline{u}_{n}\right\}_{n \in \mathbb{N}}$ and $\left\{t^{1-\alpha_{2}} \underline{v}_{n}\right\}_{n \in \mathbb{N}}$ are increasing and bounded from above. Hence the pointwise limit of $\left\{\left(t^{1-\alpha_{1}} \underline{u}_{n}, t^{1-\alpha_{2}} \underline{v}_{n}\right)\right\}_{n \in \mathbb{N}}$ exists, denoted by $\left(t^{1-\alpha_{1}} u_{*}, t^{1-\alpha_{2}} v_{*}\right)$. Hence we have

$$
\left(t^{1-\alpha_{1}} u, t^{1-\alpha_{2}} v\right)=\left(t^{1-\alpha_{1}} u_{*}, t^{1-\alpha_{2}} v_{*}\right) .
$$

Moreover, the whole sequence converges in $C([0,1]) \times C([0,1])$ to $\left(t^{1-\alpha_{1}} u_{*}, t^{1-\alpha_{2}} v_{*}\right)$. Using Lemma 2.6, we have

$$
t^{1-\alpha_{1}} \underline{u}_{n+1}(t)=a_{n, \alpha_{1}} E_{\alpha_{1}, \alpha_{1}}\left(-M_{1} t^{\alpha_{1}}\right)+t^{1-\alpha_{1}} \int_{0}^{t}(t-s)^{\alpha_{1}-1} E_{\alpha_{1}, \alpha_{1}}\left(-M_{1}(t-s)^{\alpha_{1}}\right) \widetilde{f}_{n}(s) d s,
$$

and

$$
t^{1-\alpha_{2}} \underline{v}_{n+1}(t)=b_{n, \alpha_{2}} E_{\alpha_{2}, \alpha_{2}}\left(-M_{2} t^{\alpha_{2}}\right)+t^{1-\alpha_{2}} \int_{0}^{t}(t-s)^{\alpha_{2}-1} E_{\alpha_{2}, \alpha_{2}}\left(-M_{2}(t-s)^{\alpha_{2}}\right) \widetilde{g}_{n}(s) d s
$$

where

$$
\begin{aligned}
a_{n, \alpha_{1}} & =\Gamma\left(\alpha_{1}\right) \int_{0}^{1} g_{1}(s) \underline{u}_{n}(s) d s \\
& =\Gamma\left(\alpha_{1}\right) \int_{0}^{1} g_{1}(s) s^{\alpha_{1}-1} s^{1-\alpha_{1}} \underline{u}_{n}(s) d s
\end{aligned}
$$

and

$$
\begin{aligned}
b_{n, \alpha_{2}} & =\Gamma\left(\alpha_{2}\right) \int_{0}^{1} g_{1}(s) \underline{v}_{n}(s) d s \\
& =\Gamma\left(\alpha_{2}\right) \int_{0}^{1} g_{1}(s) s^{\alpha_{2}-1} s^{1-\alpha_{2}} \underline{v}_{n}(s) d s .
\end{aligned}
$$


Letting $n \rightarrow \infty$ and $s \neq 0$, we obtain

$$
\widetilde{f}_{n}(s) \rightarrow f\left(s, u_{*}(s), v_{*}(s)\right),
$$

and

$$
\widetilde{g}_{n}(s) \rightarrow g\left(s, u_{*}(s), v_{*}(s)\right) .
$$

Also, we have

$$
\exists c_{1}>0, \forall n \in \mathbb{N}, \forall s \in[0,1],\left|s^{1-\alpha_{1}} \widehat{f}_{n}(s)\right| \leq c_{1}
$$

and

$$
\exists c_{2}>0, \forall n \in \mathbb{N}, \forall s \in[0,1],\left|s^{1-\alpha_{2}} \widehat{g}_{n}(s)\right| \leq c_{2} .
$$

Hence, the dominated convergence theorem of Lebesgue implies that

$$
t^{1-\alpha_{1}} u_{*}(t)=a_{\alpha_{1}} E_{\alpha_{1}, \alpha_{1}}\left(-M_{1} t^{\alpha_{1}}\right)+t^{1-\alpha_{1}} \int_{0}^{t}(t-s)^{\alpha_{1}-1} E_{\alpha_{1}, \alpha_{1}}\left(-M_{1}(t-s)^{\alpha_{1}}\right) \widetilde{f}(s) d s,
$$

and

$$
t^{1-\alpha_{2}} v_{*}(t)=b_{\alpha_{2}} E_{\alpha_{2}, \alpha_{2}}\left(-M_{2} t^{\alpha_{2}}\right)+t^{1-\alpha_{2}} \int_{0}^{t}(t-s)^{\alpha_{2}-1} E_{\alpha_{2}, \alpha_{2}}\left(-M_{2}(t-s)^{\alpha_{2}}\right) \widetilde{g}(s) d s,
$$

where

$$
\begin{gathered}
a_{\alpha_{1}}=\Gamma\left(\alpha_{1}\right) \int_{0}^{1} g_{0}(s) u^{*}(s) d s, \\
b_{\alpha_{2}}=\Gamma\left(\alpha_{2}\right) \int_{0}^{1} g_{2}(s) v^{*}(s) d s, \\
\widetilde{f}(s)=f\left(s, u_{*}(s), v_{*}(s)\right)+M_{1} u_{*}(s),
\end{gathered}
$$

and

$$
\widetilde{g}(s)=g\left(s, u_{*}(s), v_{*}(s)\right)+M_{2} v_{*}(s) .
$$

Using Lemma 2.6, we have

$$
\left\{\begin{array}{c}
D^{\alpha_{1}} u_{*}(t)=f\left(t, u_{*}, v_{*}\right), t \in(0,1], \\
D^{\alpha_{2}} v_{*}(t)=g\left(t, u_{*}, v_{*}\right), t \in(0,1], \\
\left.t^{1-\alpha_{1}} u_{*}(t)\right|_{t=0}=\int_{0}^{1} g_{1}(s) u_{*}(s) d s \\
\left.t^{1-\alpha_{2}} v_{*}(t)\right|_{t=0}=\int_{0}^{1} g_{2}(s) v_{*}(s) d s,
\end{array}\right.
$$

which means that $\left(u_{*}, v_{*}\right)$ is a solution of (1.1). Now, we prove that if $(u, v)$ is another solution of (1.1) such that $t^{1-\alpha_{1}} \underline{u}(t) \leq t^{1-\alpha_{1}} u(t) \leq t^{1-\alpha_{1}} \bar{u}(t)$ and $t^{1-\alpha_{2}} \underline{v}(t) \leq t^{1-\alpha_{2}} v(t) \leq t^{1-\alpha_{2}} \bar{v}(t), \forall t \in[0,1]$, then $t^{1-\alpha_{1}} u_{*}(t) \leq t^{1-\alpha_{1}} u(t)$ and $t^{1-\alpha_{2}} v_{*}(t) \leq t^{1-\alpha_{2}} v(t), \forall t \in[0,1]$. Since $(\underline{u}, \bar{u}),(\underline{v}, \bar{v})$ is a pair of lower-upper solutions of (1.1), we find from Step 3 that

$$
\forall n \in \mathbb{N}, \forall t \in[0,1], t^{1-\alpha_{1}} \underline{u}_{n}(t) \leq t^{1-\alpha_{1}} u(t) \text { and } t^{1-\alpha_{2}} \underline{v}_{n}(t) \leq t^{1-\alpha_{2}} v(t) .
$$

Letting $n \rightarrow+\infty$, we obtain

$$
\forall t \in[0,1], t^{1-\alpha_{1}} u_{*}(t) \leq t^{1-\alpha_{1}} u(t) \text { and } t^{1-\alpha_{2}} v_{*}(t) \leq t^{1-\alpha_{2}} v(t),
$$

which means that $\left(u_{*}, v_{*}\right)$ is a minimal solution of problem (1.1). The proof of Step 8 is complete.

Step 9. The sequence $\left\{\left(\bar{u}_{n}, \bar{v}_{n}\right)\right\}_{n \in \mathbb{N}}$ converges to a maximal solution $\left(u^{*}, v^{*}\right)$ of (1.1).

The proof is similar to that of Step 8, so it is omitted. Hence, the whole proof is complete. 
4. EXISTENCE OF MINIMAL- MAXIMAL AND MAXIMAL- MINIMAL SOLUTIONS FOR SYSTEMS WITH QUASIMONOTONE DECREASING FUNCTIONS

In this section, we replace the hypothesis (H3) and (H4) with the following hypothesis

(H7) $f(t, u, v)$ is decreasing in $v$ for all fixed $t \in(0,1]$, and $u \in \mathbb{R}$.

(H8) $g(t, u, v)$ is decreasing in $u$ for all fixed $t \in(0,1]$, and $v \in \mathbb{R}$.

Definition 4.1. We say that $(\underline{u}, \underline{v}),(\bar{u}, \bar{v})$ is a pair of lower-upper solutions of type 2 for problem (1.1) if

(i) $(\underline{u}, \bar{u}) \in\left(C_{1-\alpha_{1}}([0,1])\right)^{2}$ and $(\underline{v}, \bar{v}) \in\left(C_{1-\alpha_{2}}([0,1])\right)^{2}$,

$$
\begin{aligned}
& \text { (ii) }\left\{\begin{array}{l}
D^{\alpha_{1}} \underline{u}(t) \leq f(t, \underline{u}, \bar{v}), t \in(0,1] \\
D^{\alpha_{1}} \bar{u}(t) \geq f(t, \bar{u}, \underline{v}), t \in(0,1] \\
D^{\alpha_{2}} \underline{v}(t) \leq g(t, \bar{u}, \underline{v}), t \in(0,1] \\
D^{\alpha_{2}} \bar{v}(t) \geq g(t, \underline{u}, \bar{v}), t \in(0,1]
\end{array}\right. \\
& \text { (iii) }\left\{\begin{array}{l}
\left.t^{1-\alpha_{1}} \underline{u}(t)\right|_{t=0} \leq \int_{0}^{1} g_{1}(s) \underline{u}(s) d s, t \in(0,1] \\
\left.t^{1-\alpha_{1}} \bar{u}(t)\right|_{t=0} \geq \int_{0}^{1} g_{1}(s) \bar{u}(s) d s, t \in(0,1] \\
\left.t^{1-\alpha_{2}} \underline{v}(t)\right|_{t=0} \leq \int_{0}^{1} g_{1}(s) \underline{v}(s) d s, t \in(0,1] \\
\left.t^{1-\alpha_{2}} \bar{v}(t)\right|_{t=0} \geq \int_{0}^{1} g_{1}(s) \bar{v}(s) d s, t \in(0,1]
\end{array}\right.
\end{aligned}
$$

Theorem 4.2. Assume that hypothesis(H1), (H2), (Hi) for $i=5, \ldots, 8$ are satisfied and $(\underline{u}, \underline{v})$, $(\bar{u}, \bar{v})$ is a pair of lower-upper solutions of type 2 for problem (1.1) such that $t^{1-\alpha_{1}} \underline{u}(t) \leq t^{1-\alpha_{1}} \bar{u}(t)$ and $t^{1-\alpha_{2}} \underline{v}(t) \leq t^{1-\alpha_{2}} \bar{v}(t)$ in $[0,1]$. Then problem (1.1) admits a maximal-minimal solution $\left(u^{*}, v_{*}\right)$ and a minimal-maximal solution $\left(u_{*}, v^{*}\right)$ such that for every solution $(u, v)$ of $(1.1)$ with $t^{1-\alpha_{1}} \underline{u}(t) \leq t^{1-\alpha_{1}} u(t) \leq$ $t^{1-\alpha_{1}} \bar{u}(t)$ and $t^{1-\alpha_{2}} \underline{v}(t) \leq t^{1-\alpha_{2}} v(t) \leq t^{1-\alpha_{2}} \bar{v}(t)$ in $[0,1]$

$$
t^{1-\alpha_{1}} \underline{u}(t) \leq t^{1-\alpha_{1}} u_{*}(t) \leq t^{1-\alpha_{1}} u(t) \leq t^{1-\alpha_{1}} u^{*}(t) \leq t^{1-\alpha_{1}} \bar{u}(t) \text { in }[0,1]
$$

and

$$
t^{1-\alpha_{2}} \underline{v}(t) \leq t^{1-\alpha_{2}} v_{*}(t) \leq t^{1-\alpha_{2}} v(t) \leq t^{1-\alpha_{2}} v^{*}(t) \leq t^{1-\alpha_{2}} \bar{v}(t) \text { in }[0,1]
$$

Proof. We take $\bar{u}_{0}=\bar{u}, \underline{v}_{0}=\underline{v}$ and define the sequences of functions $\left\{\bar{u}_{n}\right\}_{n \geq 1},\left\{\underline{v}_{n}\right\}_{n \geq 1}$ in the following way

$$
\begin{aligned}
& \left\{\begin{array}{l}
D^{\alpha_{1}} \bar{u}_{n+1}(t)+M_{1} \bar{u}_{n+1}(t)=f\left(t, \bar{u}_{n}(t), \underline{v}_{n}(t)\right)+M_{1} \bar{u}_{n}(t), t \in(0,1], \\
\left.t^{1-\alpha_{1}} \bar{u}_{n+1}(t)\right|_{t=0}=\int_{0}^{1} g_{1}(s) \bar{u}_{n}(s) d s
\end{array}\right. \\
& \left\{\begin{array}{l}
D^{\alpha_{2}} \underline{v}_{n+1}(t)+M_{2} \underline{v}_{n}(t)=g\left(t, \bar{u}_{n}(t), \underline{v}_{n}(t)\right)+M_{2} \underline{v}_{n}(t), t \in(0,1] \\
\left.t^{1-\alpha_{2}} \underline{v}_{n}(t)\right|_{t=0}=\int_{0}^{1} g_{2}(s) \underline{v}_{n}(s) d s
\end{array}\right.
\end{aligned}
$$

Analogously, we take $\underline{u}_{0}=\underline{u}, \bar{v}_{0}=\bar{v}$ and define the sequences of functions $\left\{\underline{u}_{n}\right\}_{n \geq 1},\left\{\bar{v}_{n}\right\}_{n \geq 1}$ in the following way

$$
\begin{aligned}
& \left\{\begin{array}{l}
D^{\alpha_{1}} \underline{u}_{n+1}(t)+M_{1} \underline{u}_{n+1}(t)=f\left(t, \underline{u}_{n}(t), \bar{v}_{n}(t)\right)+M_{1} \underline{u}_{n}(t), t \in(0,1], \\
\left.t^{1-\alpha_{1}} \underline{u}_{n+1}(t)\right|_{t=0}=\int_{0}^{1} g_{1}(s) \underline{u}_{n}(s) d s
\end{array}\right. \\
& \left\{\begin{array}{l}
D^{\alpha_{2}} \bar{v}_{n+1}(t)+M_{2} \bar{v}_{n+1}(t)=g\left(t, \underline{u}_{n}(t), \bar{v}_{n}(t)\right)+M_{2} \bar{v}_{n}(t), t \in(0,1] \\
\left.t^{1-\alpha_{2}} \bar{v}_{n+1}(t)\right|_{t=0}=\int_{0}^{1} g_{2}(s) \bar{v}_{n}(s) d s
\end{array}\right.
\end{aligned}
$$

The rest of the proof is similar to that of Theorem 3.3. 


\section{EXISTENCE OF SOLUTIONS FOR SYSTEMS WITH MIXED QUASIMONOTONE FUNCTIONS}

In this section, we assume that $f(t, u, v)$ is increasing in $v$ for all fixed $t \in(0,1], u \in \mathbb{R}$ and $g(t, u, v)$ is decreasing in $u$ for all fixed $t \in(0,1], v \in \mathbb{R}$.

Definition 5.1. We say that $(\underline{u}, \underline{v}),(\bar{u}, \bar{v})$ is a pair of lower-upper solutions of type 3 for problem (1.1) if

(i) $(\underline{u}, \bar{u}) \in\left(C_{1-\alpha_{1}}([0,1])\right)^{2}$ and $(\underline{v}, \bar{v}) \in\left(C_{1-\alpha_{2}}([0,1])\right)^{2}$,

(ii)

$\left\{\begin{array}{l}D^{\alpha_{1}} \underline{u}(t) \leq f(t, \underline{u}, \underline{v}), t \in(0,1], \\ D^{\alpha_{1}} \bar{u}(t) \geq f(t, \bar{u}, \bar{v}), t \in(0,1], \\ D^{\alpha_{2}} \underline{v}(t) \leq g(t, \bar{u}, \underline{v}), t \in(0,1], \\ D^{\alpha_{2}} \bar{v}(t) \geq g(t, \underline{u}, \bar{v}), t \in(0,1],\end{array}\right.$

(iii)

$$
\left\{\begin{aligned}
\left.t^{1-\alpha_{1}} \underline{u}(t)\right|_{t=0} & \leq \int_{0}^{1} g_{1}(s) \underline{u}(s) d s \\
\left.t^{1-\alpha_{1}} \bar{u}(t)\right|_{t=0} & \geq \int_{0}^{1} g_{1}(s) \bar{u}(s) d s \\
\left.t^{1-\alpha_{2}} \underline{v}(t)\right|_{t=0} & \leq \int_{0}^{1} g_{2}(s) \underline{v}(s) d s \\
\left.t^{1-\alpha_{1}} \bar{v}(t)\right|_{t=0} & \geq \int_{0}^{1} g_{2}(s) \bar{v}(s) d s
\end{aligned}\right.
$$

Definition 5.2. The pair of functions $\left(u^{*}, v^{*}\right),\left(u_{*}, v_{*}\right)$ are called a quasisolutions of (1.1) if

(i) $\left(u^{*}, v^{*}\right) \in C_{1-\alpha_{1}}([0,1]) \times C_{1-\alpha_{2}}([0,1])$ and $\left(u_{*}, v_{*}\right) \in C_{1-\alpha_{1}}([0,1]) \times C_{1-\alpha_{2}}([0,1])$,

$$
\left\{\begin{array}{l}
D^{\alpha_{1}} u^{*}(t)=f\left(t, u^{*}, v^{*}\right), t \in(0,1], \\
D^{\alpha_{1}} u_{*}(t)=f\left(t, u_{*}, v_{*}\right), t \in(0,1], \\
D^{\alpha_{2}} v^{*}(t)=g\left(t, u_{*}, v^{*}\right), t \in(0,1], \\
D^{\alpha_{2}} v_{*}(t)=g\left(t, u^{*}, v_{*}\right), t \in(0,1], \\
\left.t^{1-\alpha_{1}} u^{*}(t)\right|_{t=0}=\int_{0}^{1} g_{1}(s) u^{*}(s) d s, \\
\left.t^{1-\alpha_{1}} u_{*}(t)\right|_{t=0}=\int_{0}^{1} g_{1}(s) u_{*}(s) d s, \\
\left.t^{1-\alpha_{2}} v^{*}(t)\right|_{t=0}=\int_{0}^{1} g_{2}(s) v^{*}(s) d s, \\
\left.t^{1-\alpha_{1}} v_{*}(t)\right|_{t=0}=\int_{0}^{1} g_{2}(s) v_{*}(s) d s .
\end{array}\right.
$$

We have the following result.

Theorem 5.3. Assume that hypothesis (Hi) for $i=1,2,3,(H 5),(H 6)$ and (H8) are satisfied and ( $\underline{u}, \underline{v})$, $(\bar{u}, \bar{v})$ is a pair of lower-upper solutions of type 3 for problem (1.1) such that $t^{1-\alpha_{1}} \underline{u}(t) \leq t^{1-\alpha_{1}} \bar{u}(t)$ and $t^{1-\alpha_{2}} \underline{v}(t) \leq t^{1-\alpha_{2}} \bar{v}(t)$ in $[0,1]$. Then problem (1.1) admits a pair of quasisolutions $\left(u^{*}, v^{*}\right),\left(u_{*}, v_{*}\right)$ such that

$$
t^{1-\alpha_{1}} \underline{u}(t) \leq t^{1-\alpha_{1}} u_{*}(t) \leq t^{1-\alpha_{1}} u^{*}(t) \leq t^{1-\alpha_{1}} \bar{u}(t) \text { in }[0,1],
$$

and

$$
t^{1-\alpha_{2}} \underline{v}(t) \leq t^{1-\alpha_{2}} v_{*}(t) \leq t^{1-\alpha_{2}} v^{*}(t) \leq t^{1-\alpha_{2}} \bar{v}(t) \text { in }[0,1]
$$

Proof. We take $u_{0}=\bar{u}, u_{1}=\underline{u}, v_{0}=\bar{v}, v_{1}=\underline{v}$ and define the sequences of functions $\left\{u_{n}\right\}_{n \geq 2}$ and $\left\{v_{n}\right\}_{n \geq 2}$ in the following way

$$
\begin{gathered}
\left\{\begin{array}{l}
D^{\alpha_{1}} u_{n+2}(t)+M_{1} u_{n+2}(t)=f\left(t, u_{n}(t), v_{n}(t)\right)+M_{1} u_{n}(t), t \in(0,1], \\
\left.t^{1-\alpha_{1}} u_{n+2}(t)\right|_{t=0}=\int_{0}^{1} g_{1}(s) u_{n}(s) d s,
\end{array}\right. \\
\left\{\begin{array}{l}
D^{\alpha_{2}} v_{n+2}(t)+M_{2} v_{n+2}(t)=f\left(t, u_{n+1}(t), v_{n}(t)\right)+M_{2} v_{n}(t), t \in(0,1], \\
\left.t^{1-\alpha_{2}} v_{n+2}(t)\right|_{t=0}=\int_{0}^{1} g_{2}(s) v_{n}(s) d s .
\end{array}\right.
\end{gathered}
$$


By using a proof, which is similar to that of Step 2 of theorem 3.3, we obtain

$$
\begin{aligned}
t^{1-\alpha_{1}} \underline{u}(t) & =t^{1-\alpha_{1}} u_{1}(t) \leq t^{1-\alpha_{1}} u_{3}(t) \leq \ldots \leq t^{1-\alpha_{1}} u_{2 n+1}(t) \leq \ldots \\
\ldots & \leq t^{1-\alpha_{1}} u_{2 n}(t) \leq \ldots \leq t^{1-\alpha_{1}} u_{2}(t) \leq t^{1-\alpha_{1}} u_{0}(t)=t^{1-\alpha_{1}} \bar{u}(t), \text { in }[0,1],
\end{aligned}
$$

and

$$
\begin{aligned}
t^{1-\alpha_{2}} \underline{v}(t) & =t^{1-\alpha_{2}} v_{1}(t) \leq t^{1-\alpha_{2}} v_{3}(t) \leq \ldots \leq t^{1-\alpha_{2}} v_{2 n+1}(t) \leq \ldots \\
\ldots & \leq t^{1-\alpha_{2}} v_{2 n}(t) \leq \ldots \leq t^{1-\alpha_{2}} v_{2}(t)=t^{1-\alpha_{2}} v_{0}(t)=t^{1-\alpha_{2}} \bar{v}(t), \text { in }[0,1]
\end{aligned}
$$

The previous inequalities show that the sequences of functions $\left\{\left(u_{2 n}, v_{2 n}\right)\right\}_{n \in \mathbb{N}}$ and $\left\{\left(u_{2 n+1}, v_{2 n+1}\right)\right\}_{n \in \mathbb{N}}$ converge to $\left(u^{*}, v^{*}\right)$ and $\left(u_{*}, v_{*}\right)$. Using a proof, which is similar to that of Step 5 of Theorem 3.3, we prove that these functions are quasisolutions of (1.1).

Since the quasisolutions are not a true solutions, it is necessary to impose additional conditions on $f$, $g$ and $g_{i}$ for $i=1,2$ which ensures that $u^{*}=u_{*}$ and $v^{*}=v_{*}$ and consequently the problem (1.1) admits at least one solution.

We assume that $\alpha_{1}=\alpha_{2}=\alpha$ and on the nonlinearities $f$ and $g$ and the functions $g_{i}$ for $i=1,2$, we shall impose the following additional conditions

(H9) There exists $M_{3}<0$ such that the function $u \longmapsto f(t, u, v)+M_{3} u$ is decreasing for all $t \in(0,1]$ and $v \in \mathbb{R}$.

(H10) There exists $M_{4}<0$ such that the function $v \longmapsto f(t, u, v)+M_{4} v$ is decreasing for all $t \in(0,1]$ and $u \in \mathbb{R}$.

(H11) There exists $M_{5}>0$ such that the function $u \longmapsto g(t, u, v)+M_{5} u$ is increasing for all $t \in(0,1]$ and $v \in \mathbb{R}$.

(H12) There exists $M_{6}<0$ such that the function $v \longmapsto g(t, u, v)+M_{6} v$ is decreasing for all $t \in(0,1]$ and $u \in \mathbb{R}$.

(H13) $\Gamma(\alpha) \int_{0}^{1} s^{\alpha-1} E_{\alpha, \alpha}\left(-M_{7} s^{\alpha_{1}}\right) g_{3}(s) d s<1$, where

$$
M_{7}=\max \left(M_{3}-M_{5}, M_{4}+M_{6}\right)
$$

and

$$
g_{3}(s)=\max \left(g_{1}(s), g_{2}(s)\right), \text { for all } s \in[0,1] .
$$

We have the following result.

Theorem 5.4. Assume that hypothesis (Hi) for $i=1,2,3,(H 5),(H 6)$ and (Hi) for $i=8, \ldots, 13$ are satisfied and $(\underline{u}, \underline{v}),(\bar{u}, \bar{v})$ is a pair of lower -upper solutions of type 3 for the problem (1.1) such that $t^{1-\alpha} \underline{u}(t) \leq$ $t^{1-\alpha} \bar{u}(t)$ and $t^{1-\alpha} \underline{v}(t) \leq t^{1-\alpha} \bar{v}(t)$ in $[0,1]$. Then problem (1.1) admits at least one solution $(u, v)$ such that $t^{1-\alpha} \underline{u}(t) \leq t^{1-\alpha} u(t) \leq t^{1-\alpha} \bar{u}(t)$ and $t^{1-\alpha} \underline{v}(t) \leq t^{1-\alpha} v(t) \leq t^{1-\alpha} \bar{v}(t)$ in $[0,1]$.

Proof. By Theorem 5.3, problem (1.1) admits a pair of quasisolutions $\left(u^{*}, v^{*}\right),\left(u_{*}, v_{*}\right)$ such that

$$
t^{1-\alpha} \underline{u}(t) \leq t^{1-\alpha} u_{*}(t) \leq t^{1-\alpha} u^{*}(t) \leq t^{1-\alpha} \bar{u}(t), \text { in }[0,1],
$$

and

$$
t^{1-\alpha} \underline{v}(t) \leq t^{1-\alpha} v_{*}(t) \leq t^{1-\alpha} v^{*}(t) \leq t^{1-\alpha} \bar{v}(t), \text { in }[0,1]
$$


Now, we put by definition

$$
\begin{gathered}
z^{*}(t)=u^{*}(t)-u_{*}(t) \text { and } w^{*}(t)=v^{*}(t)-v_{*}(t), t \in(0,1], \\
\left.t^{1-\alpha} z^{*}(t)\right|_{t=0}=\left.t^{1-\alpha} u^{*}(t)\right|_{t=0}-\left.t^{1-\alpha} u_{*}(t)\right|_{t=0},
\end{gathered}
$$

and

$$
\left.t^{1-\alpha} w^{*}(t)\right|_{t=0}=\left.t^{1-\alpha} v^{*}(t)\right|_{t=0}-\left.t^{1-\alpha} v_{*}(t)\right|_{t=0} .
$$

Using (5.1) and (5.2), we have

$$
t^{1-\alpha} z^{*}(t) \geq 0 \text { and } t^{1-\alpha} w^{*}(t) \geq 0, \text { for all } t \in[0,1] .
$$

Now, we are in a position to prove that

$$
t^{1-\alpha} z^{*}(t) \leq 0 \text { and } t^{1-\alpha} w^{*}(t) \leq 0, \text { for all } t \in[0,1] .
$$

We have

$$
\left\{\begin{array}{l}
D^{\alpha} z^{*}(t)=f\left(t, u^{*}(t), v^{*}(t)\right)-f\left(t, u_{*}(t), v_{*}(t)\right), t \in(0,1], \\
D^{\alpha} w^{*}(t)=g\left(t, u_{*}(t), v^{*}(t)\right)-g\left(t, u^{*}(t), v_{*}(t)\right), t \in(0,1], \\
\left.t^{1-\alpha} z^{*}(t)\right|_{t=0}=\int_{0}^{1} g_{1}(s) z^{*}(s) d s, \\
\left.t^{1-\alpha} w^{*}(t)\right|_{t=0}=\int_{0}^{1} g_{2}(s) w^{*}(s) d s .
\end{array}\right.
$$

From hypothesis (H9) and (H10), one has

$$
\begin{aligned}
& D^{\alpha} z^{*}(t)+M_{3} z^{*}(t)+M_{4} w^{*}(t) \\
& =f\left(t, u^{*}(t), v^{*}(t)\right)-f\left(t, u_{*}(t), v_{*}(t)\right)+M_{3} z^{*}(t)+M_{4} w^{*}(t) \\
& \leq f\left(t, u_{*}(t), v^{*}(t)\right)-f\left(t, u_{*}(t), v_{*}(t)\right)+M_{4} w^{*}(t) \\
& \leq 0
\end{aligned}
$$

that is,

$$
D^{\alpha} z^{*}(t)+M_{3} z^{*}(t)+M_{4} w^{*}(t) \leq 0, t \in(0,1] .
$$

Similarly, we find from hypothesis (H11) and (H12) that

$$
D^{\alpha} w^{*}(t)-M_{5} z^{*}(t)+M_{6} w^{*}(t) \leq 0, t \in(0,1],
$$

which implies that

$$
D^{\alpha}\left(z^{*}+w^{*}\right)(t)+M_{7} \cdot\left(z^{*}+w^{*}\right)(t) \leq 0, t \in(0,1] .
$$

Using the initial conditions in (5.4), we have

$$
\left\{\begin{array}{c}
D^{\alpha}\left(z^{*}+w^{*}\right)(t)+M_{7} \cdot\left(z^{*}+w^{*}\right)(t) \leq 0, t \in(0,1], \\
\left.t^{1-\alpha}\left(z^{*}+w^{*}\right)(t)\right|_{t=0} \leq \int_{0}^{1} g_{3}(s)\left(z^{*}+w^{*}\right)(s) d s .
\end{array}\right.
$$

From hypothesis (H13) and Lemma 2.11, we obtain

$$
t^{1-\alpha}\left(z^{*}+w^{*}\right)(t) \leq 0, \text { for all } t \in[0,1] .
$$

Consequently, by (5.3), it follows that

$$
t^{1-\alpha} z^{*}(t)=0 \text { and } t^{1-\alpha} w^{*}(t)=0 \text { for all } t \in[0,1],
$$

that is,

$$
u^{*}(t)=u_{*}(t) \text { and } v^{*}(t)=v_{*}(t) \text { for all } t \in(0,1],
$$

and

$$
\left.t^{1-\alpha} u^{*}(t)\right|_{t=0}=\left.t^{1-\alpha} u_{*}(t)\right|_{t=0} \text { and }\left.t^{1-\alpha} v^{*}(t)\right|_{t=0}=\left.t^{1-\alpha} v_{*}(t)\right|_{t=0} \text {. }
$$


It follows that problem (1.1) admits at least one solution $(u, v)$ such that $t^{1-\alpha} \underline{u}(t) \leq t^{1-\alpha} u(t) \leq t^{1-\alpha} \bar{u}(t)$ and $t^{1-\alpha} \underline{v}(t) \leq t^{1-\alpha} v(t) \leq t^{1-\alpha} \bar{v}(t)$ in $[0,1]$.

\section{EXAMPLES}

In this section, we give some examples illustrating the application of our results.

Example 6.1. We consider the following problem

$$
\left\{\begin{array}{l}
D^{\alpha_{1}} u(t)=f_{1}(t, u, v), t \in(0,1], \\
D^{\alpha_{2}} v(t)=g_{4}(t, u, v), t \in(0,1], \\
u(t)>0 \text { and } v(t)>0 \text { in }(0,1], \\
\left.t^{1-\alpha_{1}} u(t)\right|_{t=0}=\int_{0}^{1} \frac{s^{1-\alpha_{1}}}{10} u(s) d s, \\
\left.t^{1-\alpha_{2}} v(t)\right|_{t=0}=\int_{0}^{1} \frac{s^{1-\alpha_{2}}}{10} v(s) d s,
\end{array}\right.
$$

where

$$
f_{1}(t, u, v)=a_{1}(t) t^{\left(1-\alpha_{1}\right) k_{1}} u^{k_{1}}+a_{2}(t) t^{\left(1-\alpha_{2}\right) k_{2}} v^{k_{2}}+a_{3}(t) t^{\left(1-\alpha_{1}\right) k_{3}} t^{\left.1-\alpha_{2}\right) k_{4}} u^{k_{3}} v^{k_{4}}+h_{1}(t)
$$

and

$$
g_{4}(t, u, v)=b_{1}(t) t^{\left(1-\alpha_{1}\right) k_{5}} u^{k_{5}}+b_{2}(t) t^{\left(1-\alpha_{2}\right) k_{6}} v^{k_{6}}+b_{3}(t) t^{\left(1-\alpha_{1}\right) k_{7}} t^{\left(1-\alpha_{2}\right)} k_{8} u^{k_{7}} v^{k_{8}}+h_{2}(t),
$$

with $a_{i}:(0,1] \rightarrow \mathbb{R}_{+}(i=1, \ldots, 3), b_{i}:(0,1] \rightarrow \mathbb{R}_{+}(i=1, \ldots, 3)$ and $h_{i}:(0,1] \rightarrow \mathbb{R}_{+}(i=1,2)$ are continuous and bounded functions such that $t \mapsto t^{1-\alpha_{1}} a_{i}(t)(i=1, \ldots, 3), t \mapsto t^{1-\alpha_{1}} h_{1}(t), t \mapsto t^{1-\alpha_{2}} b_{i}(t)$ $(i=1, \ldots, 3), t \mapsto t^{1-\alpha_{2}} h_{2}(t)$ are continuous on $[0,1], 0<k_{i}<1$ for $i=1,2,5,6$ and $0<k_{i}+k_{i+1}<1$ for $i=3,7$.

Theorem 6.2. Problem (6.1) admits a maximal solution $\left(u^{*}, v^{*}\right)$ and a minimal solution $\left(u_{*}, v_{*}\right)$.

Proof. We put $(\underline{u}, \underline{v})=(0,0)$ and $(\bar{u}, \bar{v})=\left(L t^{\alpha_{1}-1}(1+t), L t^{\alpha_{2}-1}(1+t)\right)$, where $L$ is a positive constant. $(\underline{u}, \underline{v}),(\bar{u}, \bar{v})$ is a pair of lower-upper solutions of type 1 for problem (6.1) if

$$
\left\{\begin{array}{l}
D^{\alpha_{1}} \underline{u}(t) \leq f_{1}(t, \underline{u}, \underline{v}), t \in(0,1], \\
D^{\alpha_{1}} \bar{u}(t) \geq f_{1}(t, \bar{u}, \bar{v}), t \in(0,1], \\
D^{\alpha_{2}} \underline{v}(t) \leq g_{4}(t, \underline{u}, \underline{v}), t \in(0,1], \\
D^{\alpha_{2}} \bar{v}(t) \geq g_{4}(t, \bar{u}, \bar{v}), t \in(0,1],
\end{array}\right.
$$

and

$$
\left\{\begin{aligned}
\left.t^{1-\alpha_{1}} \underline{u}(t)\right|_{t=0} & \leq \int_{0}^{1} \frac{s^{1-\alpha_{1}}}{10} \underline{u}(s) d s, \\
\left.t^{1-\alpha_{1}} \bar{u}(t)\right|_{t=0} & \geq \int_{0}^{1} \frac{s^{1-\alpha_{1}}}{10} \bar{u}(s) d s, \\
\left.t^{1-\alpha_{2}} \underline{v}(t)\right|_{t=0} & \leq \int_{0}^{1} \frac{s^{1-\alpha_{2}}}{10} \underline{v}(s) d s, \\
\left.t^{1-\alpha_{2}} \bar{v}(t)\right|_{t=0} & \geq \int_{0}^{1} \frac{s^{1-\alpha_{2}}}{10} \bar{v}(s) d s,
\end{aligned}\right.
$$


that is,

$$
\left\{\begin{array}{l}
0 \leq h_{1}(t), t \in(0,1], \\
L \Gamma\left(\alpha_{1}+1\right) \geq a_{1}(t) L^{k_{1}}(1+t)^{k_{1}}+a_{2}(t) L^{k_{2}}(1+t)^{k_{2}} \\
\quad+a_{3}(t) L^{k_{3}+k_{4}}(1+t)^{k_{3}+k_{4}}+h_{1}(t), t \in(0,1], \\
0 \leq h_{2}(t), t \in(0,1], \\
L \Gamma\left(\alpha_{2}+1\right) \geq b_{1}(t) L^{k_{5}}(1+t)^{k_{5}}+b_{2}(t) L^{k_{6}}(1+t)^{k_{6}} \\
+b_{3}(t) L^{k_{7}+k_{8}}(1+t)^{k_{7}+k_{8}}+h_{2}(t), t \in(0,1], t \in(0,1] .
\end{array}\right.
$$

Since $0<k_{i}<1$ for $i=1,2,5,6$ and $0<k_{i}+k_{i+1}<1$ for $i=3,7$, it is not difficult to prove that if we choose $L$ such that it is sufficiently large, then $(\underline{u}, \underline{v}),(\bar{u}, \bar{v})$ is a pair of lower -upper solutions of type 1 for problem (6.1). Consequently, by Theorem 3.3, it follows that this problem admits a maximal solution $\left(u^{*}, v^{*}\right)$ and a minimal solution $\left(u_{*}, v_{*}\right)$.

Example 6.3. We consider the following problem

$$
\left\{\begin{array}{l}
D^{\alpha_{1}} u(t)=f_{2}(t, u, v), t \in(0,1] \\
D^{\alpha_{2}} v(t)=g_{5}(t, u, v), t \in(0,1] \\
u(t)>0 \text { and } v(t)>0, t \in(0,1] \\
\left.t^{1-\alpha_{1}} u(t)\right|_{t=0}=\int_{0}^{1} \frac{s^{1-\alpha_{1}}}{10} u(s) d s \\
\left.t^{1-\alpha_{2}} v(t)\right|_{t=0}=\int_{0}^{1} \frac{s^{1-\alpha_{2}}}{10} v(s) d s
\end{array}\right.
$$

where

$$
f_{2}(t, u, v)=a_{1}(t) t^{\left(1-\alpha_{1}\right) k_{1}} u^{k_{1}}-a_{2}(t) t^{\left(1-\alpha_{2}\right) k_{2}} v^{k_{2}}+h_{1}(t),
$$

and

$$
g_{5}(t, u, v)=-b_{1}(t) t^{\left(1-\alpha_{1}\right) k_{5}} u^{k_{5}}+b_{2}(t) t^{\left(1-\alpha_{2}\right) k_{6}} v^{k_{6}}+h_{2}(t)
$$

with $a_{i}:(0,1] \rightarrow \mathbb{R}_{+}(i=1,2), b_{i}:(0,1] \rightarrow \mathbb{R}_{+}(i=1,2)$ and $h_{i}:(0,1] \rightarrow \mathbb{R}_{+}(i=1,2)$ are continuous and bounded functions such that $t \mapsto t^{1-\alpha_{1}} a_{i}(t)$ (i=1,2), $t \mapsto t^{1-\alpha_{1}} h_{1}(t), t \mapsto t^{1-\alpha_{2}} b_{i}(t)(i=1,2)$, $t \mapsto t^{1-\alpha_{2}} h_{2}(t)$ are continuous on $[0,1], 0<k_{i}<1$ for $i=1,2,5,6$.

Theorem 6.4. Problem (6.2) admits a maximal-minimal solution $\left(u^{*}, v_{*}\right)$ and a minimal-maximal solution $\left(u_{*}, v^{*}\right)$.

Proof. We put $(\underline{u}, \underline{v})=(0,0)$ and $(\bar{u}, \bar{v})=\left(L_{1} t^{\alpha_{1}-1}(1+t), L_{2} t^{\alpha_{2}-1}(1+t)\right)$, where $L_{1}$ and $L_{2}$ are two positive real numbers. $(\underline{u}, \underline{v}),(\bar{u}, \bar{v})$ is a pair of lower-upper solutions of type 2 for problem (6.2) if

$$
\left\{\begin{array}{l}
D^{\alpha_{1}} \underline{u}(t) \leq f_{2}(t, \underline{u}, \bar{v}), t \in(0,1], \\
D^{\alpha_{1}} \bar{u}(t) \geq f_{2}(t, \bar{u}, \underline{v}), t \in(0,1], \\
D^{\alpha_{2}} \underline{v}(t) \leq g_{5}(t, \bar{u}, \underline{v}), t \in(0,1], \\
D^{\alpha_{2}} \bar{v}(t) \geq g_{5}(t, \underline{u}, \bar{v}), t \in(0,1],
\end{array}\right.
$$


and

$$
\left\{\begin{aligned}
\left.t^{1-\alpha_{1}} \underline{u}(t)\right|_{t=0} & \leq \int_{0}^{1} \frac{s^{1-\alpha_{1}}}{10} \underline{u}(s) d s, \\
\left.t^{1-\alpha_{1}} \bar{u}(t)\right|_{t=0} & \geq \int_{0}^{1} \frac{s^{1-\alpha_{1}}}{10} \bar{u}(s) d s, \\
\left.t^{1-\alpha_{2}} \underline{v}(t)\right|_{t=0} & \leq \int_{0}^{1} \frac{s^{1-\alpha_{2}}}{10} \underline{v}(s) d s, \\
\left.t^{1-\alpha_{2}} \bar{v}(t)\right|_{t=0} & \geq \int_{0}^{1} \frac{s^{1-\alpha_{2}}}{10} \bar{v}(s) d s,
\end{aligned}\right.
$$

that is,

$$
\left\{\begin{array}{l}
0 \leq-a_{2}(t) L_{2}^{k_{2}}(1+t)^{k_{2}}+h_{1}(t), t \in(0,1], \\
L_{1} \Gamma\left(\alpha_{1}+1\right) \geq a_{1}(t) L_{1}^{k_{1}}(1+t)^{k_{1}}+h_{1}(t), t \in(0,1], \\
0 \leq-b_{1}(t) L_{1}^{k_{5}}(1+t)^{k_{5}}+h_{2}(t), t \in(0,1], \\
L_{2} \Gamma\left(\alpha_{2}+1\right) \geq b_{2}(t) L_{2}^{k_{6}}(1+t)^{k_{6}}+h_{2}(t), t \in(0,1], t \in(0,1] .
\end{array}\right.
$$

Put

$$
\begin{gathered}
C_{1}=\max \left(1, \sup _{t \in(0,1]} \frac{h_{1}(t)}{\Gamma\left(\alpha_{1}+1\right)-a_{1}(t)(1+t)^{k_{1}}}\right), \\
C_{2}=\inf _{t \in(0,1]}\left(\frac{h_{2}(t)}{b_{1}(t)(1+t)^{k_{5}}}\right)^{\frac{1}{k_{5}}}, \\
C_{3}=\max \left(1, \sup _{t \in(0,1]} \frac{h_{2}(t)}{\Gamma\left(\alpha_{2}+1\right)-b_{2}(t)(1+t)^{k_{6}}}\right),
\end{gathered}
$$

and

$$
C_{4}=\inf _{t \in(0,1]}\left(\frac{h_{1}(t)}{a_{2}(t)(1+t)^{k_{2}}}\right)^{\frac{1}{k_{2}}} .
$$

If we suppose that the constants $C_{i}(i=1, \ldots, 4)$ are well defined, $C_{1} \leq C_{2}$ and $C_{3} \leq C_{4}$ and $C_{1} \leq L_{1} \leq C_{2}$ and $C_{3} \leq L_{2} \leq C_{4}$, then $(\underline{u}, \underline{v}),(\bar{u}, \bar{v})$ is a pair of lower -upper solutions of type 2 for problem (6.2). Using Theorem 4.2, we find that that this problem admits a maximal-minimal solution $\left(u^{*}, v_{*}\right)$ and a minimal-maximal solution $\left(u_{*}, v^{*}\right)$.

Example 6.5. We consider the following problem

$$
\left\{\begin{array}{l}
D^{\alpha} u(t)=f_{3}(t, u, v), t \in(0,1] \\
D^{\alpha} v(t)=g_{6}(t, u, v), t \in(0,1] \\
u(t)>0 \text { and } v(t)>0, t \in(0,1] \\
\left.t^{1-\alpha} u(t)\right|_{t=0}=\frac{1}{\Gamma(\alpha) E_{\alpha, \alpha}(A)} \int_{0}^{1} \frac{s^{1-\alpha}}{10} u(s) d s \\
\left.t^{1-\alpha} v(t)\right|_{t=0}=\frac{1}{\Gamma(\alpha) E_{\alpha, \alpha}(B)} \int_{0}^{1} \frac{s^{1-\alpha}}{10} v(s) d s
\end{array}\right.
$$

where

$$
f_{3}(t, u, v)=a_{1}(t) t^{1-\alpha_{1}} u+a_{2}(t) t^{\left(1-\alpha_{2}\right) k_{2}} v^{k_{2}}+h_{1}(t)
$$

and

$$
g_{6}(t, u, v)=-b_{1}(t) t^{1-\alpha_{1}} u+b_{2}(t) t^{1-\alpha_{2}} v+h_{2}(t),
$$


with $a_{i}:(0,1] \rightarrow \mathbb{R}_{+}(i=1,2), b_{i}:(0,1] \rightarrow \mathbb{R}_{+}(i=1,2)$ and $h_{i}:(0,1] \rightarrow \mathbb{R}_{+}(i=1,2)$ are continuous and bounded functions such that $t \mapsto t^{1-\alpha} a_{i}(t)(i=1,2), t \mapsto t^{1-\alpha} h_{i}(t)(i=1,2), t \mapsto t^{1-\alpha} b_{i}(t)$ $(i=1,2)$ are continuous on $[0,1], 0<k_{2}<1, A=\max _{t \in[0,1]}\left|a_{1}(t) t^{1-\alpha}\right|$ and $B=\max _{t \in[0,1]}\left|b_{2}(t) t^{1-\alpha}\right|$.

Theorem 6.6. Problem (6.3) admits at least one solution a solution $(u, v)$.

Proof. We put $(\underline{u}, \underline{v})=(0,0)$ and $(\bar{u}, \bar{v})=\left(L t^{\alpha-1}(1+t), L t^{\alpha-1}(1+t)\right)$, where $L$ is a positive constant. $(\underline{u}, \underline{v}),(\bar{u}, \bar{v})$ is a pair of lower-upper solutions of type 3 for problem (6.3) if

$$
\left\{\begin{array}{l}
D^{\alpha} \underline{u}(t) \leq a_{1}(t) t^{1-\alpha} \underline{u}+a_{2}(t) t^{(1-\alpha) k_{2}} \underline{v}^{k_{2}}+h_{1}(t), t \in(0,1], \\
D^{\alpha} \bar{u}(t) \geq a_{1}(t) t^{1-\alpha} \bar{u}+a_{2}(t) t^{(1-\alpha) k_{2}} \bar{v}^{k_{2}}+h_{1}(t), t \in(0,1], \\
D^{\alpha} \underline{v}(t) \leq-b_{1}(t) t^{1-\alpha} \bar{u}+b_{2}(t) t^{1-\alpha} \underline{v}+h_{2}(t), t \in(0,1], \\
D^{\alpha} \bar{v}(t) \geq-b_{1}(t) t^{1-\alpha} \underline{u}+b_{2}(t) t^{1-\alpha} \bar{v}+h_{2}(t), t \in(0,1],
\end{array}\right.
$$

and

that is,

$$
\left\{\begin{aligned}
\left.t^{1-\alpha} \underline{u}(t)\right|_{t=0} & \leq \frac{1}{\Gamma(\alpha) E_{\alpha, \alpha}(A)} \int_{0}^{1} \frac{s^{1-\alpha}}{10} \underline{u}(s) d s, \\
\left.t^{1-\alpha} \bar{u}(t)\right|_{t=0} & \geq \frac{1}{\Gamma(\alpha) E_{\alpha, \alpha}(A)} \int_{0}^{1} \frac{s^{1-\alpha}}{10} \bar{u}(s) d s, \\
\left.t^{1-\alpha} \underline{v}(t)\right|_{t=0} & \leq \frac{1}{\Gamma(\alpha) E_{\alpha, \alpha}(B)} \int_{0}^{1} \frac{s^{1-\alpha}}{10} \underline{v}(s) d s, \\
\left.t^{1-\alpha} \bar{v}(t)\right|_{t=0} & \geq \frac{1}{\Gamma(\alpha) E_{\alpha, \alpha}(B)} \int_{0}^{1} \frac{s^{1-\alpha}}{10} \bar{v}(s) d s,
\end{aligned}\right.
$$

$$
\left\{\begin{array}{l}
0 \leq h_{1}(t), t \in(0,1] \\
L \Gamma(\alpha+1) \geq a_{1}(t) L(1+t)+a_{2}(t) L^{k_{2}}(1+t)^{k_{2}}+h_{1}(t), t \in(0,1], \\
0 \leq-b_{1}(t) L(1+t)+h_{2}(t), t \in(0,1], \\
L \Gamma(\alpha+1) \geq b_{2}(t) L(1+t)+h_{2}(t), t \in(0,1], t \in(0,1] .
\end{array}\right.
$$

If we suppose that the following constants

$$
\begin{gathered}
\widetilde{C_{5}}=\sup _{t \in(0,1]} \frac{h_{1}(t)}{\Gamma(\alpha+1)-a_{1}(t)(1+t)-a_{2}(t)(1+t)^{k_{2}}}, \\
\widehat{C_{5}}=\sup _{t \in(0,1]} \frac{h_{2}(t)}{\Gamma(\alpha+1)-b_{2}(t)(1+t)},
\end{gathered}
$$

and

$$
C_{6}=\inf _{t \in(0,1]} \frac{h_{2}(t)}{b_{1}(t)(1+t)},
$$

are well defined and

$$
C_{5}:=\max \left(1, \widetilde{C_{5}}, \widehat{C_{5}}\right) \leq C_{6},
$$

and if we choose $C_{5} \leq L \leq C_{6}$, then $(\underline{u}, \underline{v}),(\bar{u}, \bar{v})$ is a pair of lower -upper solutions of type 3 for problem (6.3). Consequently, we find from Theorem 5.4 that this problem admits at least a solution $(u, v)$ such that $t^{1-\alpha} \underline{u}(t) \leq t^{1-\alpha} u(t) \leq t^{1-\alpha} \bar{u}(t)$ and $t^{1-\alpha} \underline{v}(t) \leq t^{1-\alpha} v(t) \leq t^{1-\alpha} \bar{v}(t)$ in $[0,1]$.

\section{Acknowledgements}

The authors are grateful to the reviewers for several comments and suggestions which contributed to improve this paper. This work was supported by a MESRS-DRS grant C00L03UN13012014112. 
M. DERHAB, F. MEZIANE

\section{REFERENCES}

[1] W. M. Ahmad, R. El-Khazali, Fractional-order dynamical models of love, Chaos Solitons Fractals 33 (2007), 1367-1375.

[2] R. L. Bagley, P. J. Torvik, On the fractional calculus model of viscoelastic behavior, J. Rheol. 30 (1986), 133-155.

[3] A. Dokoumetzidis, Richard Magin, P. Macheras, Fractional kinetics in multi-compartmental systems, J. Pharmacokinet Pharmacodyn. 37 (2010), 507-524.

[4] A. A. Kilbas, H.M. Srivastava, J.J. Trujillo, Theory and Applications of Fractional Differential Equations. North-Holland Mathematics Studies, 204, Elsevier Science B.V., Amsterdam, 2006.

[5] S.G. Samko, A.A. Kilbas, O.I. Marichev, Fractional integrals and derivatives: theory and applications, Gordon and Breach, New York, 1993.

[6] I. Podlubny, Fractional Differential Equations, Academic Press, San Diego, 1999.

[7] B. Ahmad, J. J. Nieto, Existence results for a coupled system of nonlinear fractional differential equations with three-point boundary conditions, Comput. Math. Appl. 58 (2009), 1838-1843.

[8] J. Henderson, R. Luca, Boundary Value Problems for Systems of Differential, Difference and Fractional Equations: Positive Solutions, Elsevier, Amsterdam, 2016.

[9] J. Henderson, R. Luca, A. Tudorache, On a system of fractional differential equations with coupled integral boundary conditions, Fract. Calc. Appl. Anal. 18 (2015), 361-386.

[10] L. Zhang, B. Ahmad, G. Wang, The existence of an extremal solution to a nonlinear system with the right-handed Riemann-Liouville fractional derivative, Appl. Math. Lett. 31 (2014), 1-6.

[11] Z. Bai, S. Zhang, S. Sun, C. Yin, Monotone iterative method for fractional differential equations, Electron. J. Differential Equations 2016 (2016), 1-8.

[12] M. Delgado, A. Suárez, Existence of solutions for elliptic systems with Hölder continuous nonlinearities, Differential Integral Equations 13 (2000), 453-477.

[13] Z. Denton, A. S. Vatsala, Monotone iterative technique for finite systems of nonlinear Riemann-Liouville fractional differential systems, Opuscula Math. 31 (2011), 327-339.

[14] M. Derhab, A quasilinear elliptic system with integral boundary conditions, Proc. Jangjeon Math. Soc. 12 (2009), $165-187$.

[15] V. Lakshmikantham, S. Leela, J. Vasundhara, Theory of Fractional Dynamic Systems, Cambridge Academic Publishers, Cambridge, 2009.

[16] C. V. Pao, Nonlinear parabolic and elliptic equations, Plenum Press, New York, 1992.

[17] G. Wang, R. P. Agarwal, A. Cabada, Existence results and the monotone iterative technique for systems of nonlinear fractional differential equations, Appl. Math. Lett. 25 (2012), 1019-1024.

[18] J. H. Barrett, Differential equations of non-integer order, Canad. J. Math. 6 (1954), 529-541.

[19] C. Kou, H. Zhou, C. Li, Existence and continuation theorems of Riemann-Liouville type fractional differential equations, Int. J. Bifurc. Chaos 22 (2012), Article ID 1250077. 